\title{
Original
}

\section{Satisfacción Laboral y Apoyo Social en trabajadores de un hospital de tercer nivel}

\section{Job satisfaction and perceived social support among workers from a tertiary hospital}

\author{
Marco Gandarillas González ', Luis Vásquez Rueda ', Héctor Márquez Marmolejo ', Eva Santamaría Gandarillas ', \\ Óscar Garaña Robles ', Miguel Santibáñez Margüello 1,2 \\ 1. Hospital Marqués de Valdecilla. Unidad Docente de Medicina del Trabajo de Cantabria. Consejería de Salud \\ y Servicios Sociales. Gobierno de Cantabria. España. \\ 2. Universidad de Cantabria. Departamento de Enfermería. Instituto de Investigación de Valdecilla (IDIVAL). Grupo \\ de Investigación en Salud Pública.
}

Recibido: 08-01-14

Aceptado: 24-02-14

\author{
Correspondencia: \\ Dr. Marco Gandarillas González. \\ Hospital Universitario Marqués de Valdecilla \\ Avenida de Valdecilla s/n \\ Santander. España \\ Tfno. 9422025 20. Fax 942315446 \\ Email: magandarillas@humv.es.
}

Resumen

Objetivo: Realizar una evaluación del grado de satisfacción laboral y de apoyo social percibido en profesionales del Hospital Universitario Marqués de Valdecilla (HUMV).

Trabajadores y método: Estudio transversal, descriptivo y analítico, en respondedores a un cuestionario con variables sociodemográficas, de la escala de satisfacción laboral de Warr, Cook y Wall (1979), y de la escala de valoración del apoyo social percibido de House y Wells (1978), ambas validadas por el INSHT. El cuestionario se puso a disposición de todos los profesionales, la respuesta fue voluntaria y se preservó el anonimato.

Resultados: Respondieron 676 trabajadores, $14,6 \%$ de la plantilla. La satisfacción general percibida fue en grado neutro. Se encontró diferencia por ámbito laboral $(p=0,005)$, edad $(p=0,01)$ y estado civil $(p=0,01)$. Los factores mejor apreciados fueron la relación con compañeros, supervisor directo, responsabilidad asignada, variedad de tareas, estabilidad en el empleo y horario. Especialmente insatisfactorias resultaron salario, atención a sugerencias, posibilidades de promoción y modo de gestión de la organización.

Se observó mayor satisfacción con la tarea a realizar en facultativos, DUE y técnicos de laboratorio (TL), y menor en auxiliares de enfermería y celadores $(\mathrm{p}<0,00005)$. Por edad fue menor en el grupo de 31-40 años $(\mathrm{p}=0,05)$. Las satisfacción con las condiciones en que desarrollan su tarea fue asimismo mayor en facultativos, DUE y TL que en auxiliares de enfermería y celadores $(p<0,00005)$; y por ámbito laboral mayor en consultas, plantas y bloque quirúrgico que en servicios centrales, UCIs y Urgencias $(\mathrm{p}=0,004)$. La satisfacción con las personas de la organización se asoció al ámbito laboral $(\mathrm{p}<0,00005)$, profesión $(\mathrm{p}=0,001)$ $y$ edad $(p=0,005)$.

El apoyo instrumental en el trabajo se correlacionó con la satisfacción con el superior jerárquico $(\mathrm{r}=0,77 ; \mathrm{p}<0,00005)$ y con las relaciones con la Dirección $(\mathrm{r}=0,70 ; \mathrm{p}<0,00005)$. El sanitario percibió mayor apoyo instrumental que el no sanitario $(\mathrm{p}=0,01)$ y por lugar de trabajo fue menor la percepción en servicios centrales $(\mathrm{p}<0,00005)$. 
El apoyo emocional en el trabajo mostró fuerte correlación con la satisfacción con el superior jerárquico $(r=0,75 ; \mathrm{p}<0,00005)$ y relaciones con la Dirección $(r=0,69 ; \mathrm{p}<0,00005)$. De segundo orden fue la correlación con la satisfacción con compañeros, el reconocimiento y el uso de la capacidad profesional.

Se observó fuerte correlación entre la satisfacción con las condiciones de trabajo y la satisfacción con la tarea realizada $(r=0,84 ; \mathrm{p}<0,00005)$. Así mismo, se encontró fuerte correlación entre la satisfacción general, con la tarea, con las condiciones de trabajo, con las personas y con el apoyo emocional e instrumental en el trabajo. El análisis multivariante mostró una relación independiente entre la satisfacción laboral general y el apoyo en el trabajo emocional ( $p=0,0001$; OR: 1,16-1,61) e instrumental ( $p=0,0018$, OR: 1,15-1,97).

Conclusiones: La satisfacción general es en promedio de grado neutro, si bien hay diferencia por ámbito laboral, edad y estado civil. Los tres factores mejor valorados fueron compañeros, responsabilidad asignada y estabilidad en el empleo. Los tres peores salario, atención a sugerencias y modo de gestión de la organización. El apoyo en el trabajo emocional e instrumental fueron las variables independientes asociadas al grado de satisfacción laboral. Deben potenciarse estos aspectos y mejorar los factores de satisfacción peor valorados.

Med Segur Trab (Internet) 2014; 60 (234) 64-89

Palabras clave: Satisfacción laboral. Apoyo social. Administración Hospitalaria.

Abstract

Objective: To evaluate the perception of satisfaction and perceived social support in the workplace among staff of University Hospital Marqués de Valdecilla.

Workers and methodology: Descriptive and analytical, cross-sectional study among responders who selfcompleted a questionnaire about sociodemographic variables, and about the items from the questionnaires specifically developed by Warr, Cook y Wall (1979) 'Job satisfaction: Overall Job Satisfaction Scale' and House y Wells (1978) 'Social support', both validated by the INHT. Questionnaire was available for all workers, the response was voluntary and anonymity was preserved.

Results: 676 workers completed the questionnaire, $14.6 \%$ out of the whole staff. Perceived overall Job Satisfaction was neutral. Statistically significant diffferences were found according to occupational area $(p=0.005)$, age $(p=0.01)$ and civil status $(p=0.01)$. The best valorated elements were the relationship with workmates, direct supervisor, assigned responsibility, taks variety, job security and timetable. Salary, feedback to suggestions, promotion possibilities, and the way of organization management were specially unsatisfactory.

A higher satisfaction regarding tasks to perform was observed among doctors, nursery and laboratory staff; and a lower satifastion was observed among nursery assistants and porters $(\mathrm{p}<0.00005)$. According to age, satisfaction was lower among 31-40 years old $(\mathrm{p}=0.05)$. Regarding the work conditions in which such tasks are performed, a higher satisfaction was also observed among doctors, nursery and laboratory staff in contrast to nursery assistants and porters $(\mathrm{p}<0.00005)$. In relation to work setting, satisfaction was higher in consulting rooms, hospitalization floors, and surgery area with respect to central offices, ICUs and Emergency $(\mathrm{p}=0.004)$. Satisfaction with people at organization was associated to work setting $(\mathrm{p}<0.00005)$, occupation (job tittle) $(\mathrm{p}=0.001)$ and age $(\mathrm{p}=0.005)$.

Instrumental support at work showed a strong correlation in relation to satisfaction with the superior hierarchical level supervisor $(r=0.77 ; \mathrm{p}<0.00005)$ and relationship with executive management $(\mathrm{r}=0.70 ; \mathrm{p}<0.00005)$. Healthcare staff perceived a higher instrumental support than Non-Healthcare staff $(\mathrm{p}=0.01)$. Instrumental support was lower in central offices $(\mathrm{p}<0.00005)$.

Emotional support at work showed also a strong correlation in relation to satisfaction with the superior hierarchical level supervisor $(r=0.75 ; \mathrm{p}<0.00005)$ and relationship with executive management $(\mathrm{r}=0.69 ; \mathrm{p}<0.00005)$. Less strong correlations were found in relation to satisfaction with workmates, recognition and use of proffessional capacitation.

A strong correlation was observed between work conditions and satisfaction regarding tasks to perform ( $r=0.84 ; \mathrm{p}<0.00005)$. Likewise, strong correlations were found between overall Job Satisfaction, satisfaction regarding tasks to perform, work conditions, workmates, and emotional and instrumental support at work. Multivariable analysis showed an independent association between overall Job Satisfaction and emotional $(\mathrm{p}=0.0001$; OR: 1.16-1.61) and instrumental support $(\mathrm{p}=0.0018, \mathrm{OR}: 1.15-1.97)$. 
Conclusions: Overall Job Satisfaction was on average neutral, but there were differences according to occupational area, age and civil status. The three best valorated elements were relationship with workmates, assigned responsibility, and job security. The three worse valorated ones were salary, feedback to suggestions, and the way of organization management. Intrumental and emotional support were independent variables associated with job satisfaction. These aspects must be strengthened whereas worse valorated elements must be improved.

Med Segur Trab (Internet) 2014; 60 (234) 64-89

Keywords: Job Satisfaction. Social Support. Hospital Administration. 


\section{INTRODUCCIÓN}

La evaluación de los factores psicosociales de las condiciones de trabajo viene impuesto por la normativa preventiva ${ }^{1,2}$, y a este respecto el estudio de la satisfacción laboral (SL) tiene interés especial ${ }^{3}$. La SL indica la capacidad de una Organización para satisfacer las necesidades de sus trabajadores en los aspectos psicosociales y predice ciertas pautas de comportamiento disfuncional en los mismos. La SL se relaciona con la motivación y el clima laboral ${ }^{4}$, se ha convertido en un tema central de investigación en psicosociología de la organización y ordenación del trabajo, y es uno de los factores de la calidad de vida laboral que capta mayor interés en prevencionistas y gestores del ámbito sanitario 5 .

Los profesionales insatisfechos tienden a ver resentida su salud psicofísica por alteración del estado de ánimo y diversas quejas sicosomáticas, además de ser menos eficientes, de mayor absentismo y demanda de cambio de puesto de trabajo. Un elevado índice de insatisfacción produce un decremento en la eficiencia global de la organización, a través de conductas agresivas de maledicencia, negligencia, sabotaje y con tendencia al abandono del profesional por no encontrar un puesto acorde a sus expectativas ${ }^{6,7}$. Por otro lado, la SL es un factor determinante de la retención del trabajador y se sabe que para éste el valor de su puesto de trabajo puede cambiar en la medida en que cambia su grado de SL en el mismo ${ }^{8}$.

No se dispone de una definición unánime de la SL y se suele considerar como el balance percibido sobre cuánto y cómo se acomodan las características del puesto de trabajo a las expectativas del trabajador. Del balance puede resultar una orientación afectiva positiva o negativa hacia el puesto. También se ha definido como un estado emocional positivo que resulta de una percepción placentera de la experiencia laboral ${ }^{3}$. Los aspectos de SL más mencionados se relacionan tanto con el contenido de la tarea como grado de interés, reto, desafío, variedad u oportunidad de aprendizaje, como con las condiciones ajenas a la misma como salario, horario y condiciones físicas o ambientales.

Ante la variedad aspectos a valorar Herzberg propuso su teoría de dos conjuntos de factores del trabajo bien diferenciados y a la vez relacionados con la SL: "factores de intrínsecos o motivadores" y "factores de extrínsecos o higiénicos" ${ }^{\text {. Son factores }}$ intrínsecos a la tarea posibilidad de desarrollo personal, interés de la tarea, logro, reconocimiento, creatividad y tener responsabilidad y posibilidad de promoción. Se consideran aspectos extrínsecos a la tarea los relacionados con la política de la organización, calidad de supervisión, relaciones con compañeros, salario y condiciones físicas o ambientales. Herzberg apreció que sólo originan SL los elementos intrínsecos, mientras que los que producen insatisfacción son los extrínsecos. Otros rasgos del trabajador como edad, educación, género y circunstancias no laborales también han recibido atención como moduladoras de la SL.

El concepto de apoyo social también despierta interés en psicosociología laboral ${ }^{9}$, y se le considera un factor moderador del efecto sobre la salud psicofísica de los estresores laborales inevitables, al satisfacer la necesidad del trabajador de ayuda, pertenencia y estima ${ }^{10}$.

En el ámbito sanitario el estudio de la SL y los factores asociados han sido motivo de estudio ${ }^{11-16}$. Sin embargo el apoyo social percibido y los factores que lo condicionan han sido menos evaluados, y menos aún la relación entre la SL y el apoyo social. El presente estudio pretende evaluar el grado de SL en profesionales en un Hospital Universitario, e identificar los factores asociados tanto laborales como no laborales. Así mismo, valorar el apoyo social en el ámbito laboral y no laboral y su relación con la SL.

\section{TRABAJADORES Y MÉTODO}

Se realizó un estudio de transversal, descriptivo y analítico, para evaluar el grado de SL general, apoyo social percibido y variables relacionadas en profesionales del Hospital 
Universitario Marqués de Valdecilla (HUMV). Es un hospital de tercer nivel del Servicio Cántabro de Salud con una plantilla media estimada de 4600 trabajadores en el período del estudio, con mediana de edad de 50 años y $80 \%$ del género femenino.

La fuente de obtención de datos fue de tipo primario mediante un cuestionario autocumplimentado aplicado de forma colectiva para facilitar la recogida eficiente de la información. Los cuestionarios se dispusieron de forma contemporánea en marzo de 2013 en los diversos centros dependientes del HUMV y su cumplimentación fue voluntaria. Con el fin de asegurar la confidencialidad el cuestionario se diseñó de forma que su cumplimentación fuera anónima y se utilizaron buzones opacos para la recogida.

\section{Satisfacción laboral general}

Para evaluar la SL general se empleó el cuestionario de Warr, Cook y Wall (1979), cuya estructura se muestra en el anexo 1 (Pérez y Fidalgo, NTP 394, INSHT 1994). Se basa en los factores dicotómicos intrínsecos y extrínsecos de Herzberg. El cuestionario se cumplimenta en unos minutos y consta de 15 items cuya respuesta se da en una escala descriptiva discontinua de Likert que atribuye adjetivos a los distintos puntos del continuo (p.e. ¿Grado de satisfacción con el ambiente de trabajo?: Muy insatisfecho-InsatisfechoPoco insatisfecho-Neutro-Poco Satisfecho-Satisfecho-Muy satisfecho). Todos los ítems son variables cualitativas ordinales de respuesta única. La puntuación de SL general se obtiene de forma aditiva, sumando las puntuaciones dadas en cada uno de los 15 ítems, asignando el valor de 1 a Muy insatisfecho y correlativamente hasta asignar el valor de 7 a Muy satisfecho. En consecuencia para la SL general la puntuación total oscila entre 15 y 105, y a mayor puntuación mayor satisfacción.

\section{Satisfacción extrínseca, satisfacción intrínseca y satisfacción con las personas}

El cuestionario de SL general permite a su vez generar dos subescalas. Así, se crearon las subescalas de satisfacción laboral intrínseca y de satisfacción extrínseca propuestas por Warr, Cook y Wall basándose en la teoría de Herzberg.

La subescala de factores intrínsecos aborda aspectos relativos al contenido de la tarea como el reconocimiento laboral, responsabilidad y promoción. Está formada por siete ítems de la escala general: 2, 4, 6, 8, 10, 12 y 14. Su corrección es idéntica a la de la escala general si bien debido a su menor longitud sus valores oscilan entre 7 y 49 .

La subescala de factores extrínsecos aborda aspectos relativos a la organización del trabajo como el horario, el salario o las condiciones físicas del puesto. Esta subescala lo constituyen ocho ítems: 1, 3, 5, 7, 9, 11, 13 y 15. Su corrección idéntica a la de la escala general, si bien debido a su menor longitud su rango de valores oscila entre 8 y 56 .

Además, y acorde con otros autores, se consideró de interés valorar una tercera subescala que tratara el grado SL con las personas de la Organización: compañeros, supervisores y Dirección. Esta subescala se corresponde con la suma de los ítems 3, 4, 5, 9, 11 y 12. Su rango de valores oscila entre 6 y 42 .

\section{Escala de apoyo social}

Para evaluar el apoyo social se empleó la escala de House y Wells (1978), cuya estructura se muestra en el anexo 2 (Pérez y Martín, NTP 439; INSHT). La escala distingue entre apoyo social emocional e instrumental procedente de diversas fuentes. Es un cuestionario de aplicación individual e identifica las principales fuentes de apoyo social laboral y extralaboral para el trabajador. El apoyo emocional laboral se valora por los items a1, b1, a2, b2 y b4. El apoyo emocional no laboral por los ítems c1, d1, c2 y d2. Y el apoyo instrumental laboral por los items a3, b3 y a4. La puntuación de cada bloque se obtiene por la suma de los valores de sus ítems. Así, la puntuación máxima es de 15 para el apoyo emocional laboral, 12 para el emocional no laboral y 9 para el apoyo instrumental laboral. 


\section{Método estadístico}

Las variables cualitativas se describen en frecuencia absoluta y relativa, las continuas por su media o mediana y rango. Para estudiar la relación entre variables cualitativas se empleó la $\chi^{2}$, entre cualitativas y cuantitativas la $\mathrm{t}$ de Student o el ANOVA con el test postprueba de Scheffe, y entre cuantitativas regresión lineal con el coeficiente de correlación lineal de Pearson. Cuando fue necesario se aplicaron las pruebas no paramétricas equivalentes de Mann-Whytney, Kruskall-Wallis y el coeficiente de correlación ordinal de Spearman. La hipótesis de normalidad se comprobó con la prueba de Kolmogorov-Smirnov, y la de concordancia en la valoración de factores con la W de Kendall. Para identificar las variables asociadas independientes con la escala de SL se empleó regresión logística por el método de selección paso a paso hacia delante. Se consideró significativa la asociación con un error $\alpha(\mathrm{p})<0,05$. El análisis se realizó con el paquete estadístico SPSS v15.

\section{RESULTADOS}

\section{Descripción de la muestra}

Respondieron la encuesta 696 profesionales, 15,1\% de la plantilla media. Se descartaron 19 cuestionarios por deficiente cumplimentación, resultando objeto de análisis final $676,14,6 \%$ de la plantilla. Un 80\% fueron mujeres, la mediana de edad 49 años, la de antigüedad en la profesión 21 años, y la de antigüedad como trabajador del HUMV 17 años. Las siguientes tablas describen la distribución por profesión, lugar de trabajo, estado civil, tipo de contrato y número de hijos.

\begin{tabular}{lrr}
\multicolumn{3}{c}{ Profesión } \\
\hline & N & $\%$ \\
\hline MÉDICO & 82 & 12 \\
DUE & 297 & 45 \\
AUX. ENF. & 178 & 27 \\
CELADOR & 43 & 7 \\
ADMIN. & 34 & 5 \\
TEL. & 8 & 1 \\
OTRA & 16 & 2 \\
Total & 658 & 100 \\
Total & 18 & \\
\hline
\end{tabular}

\begin{tabular}{lrr}
\multicolumn{3}{c}{ Ámbito laboral } \\
\hline & N & $\%$ \\
\hline Plantas & 299 & 46 \\
Consultas & 67 & 10 \\
Urgencias/UCIs & 82 & 13 \\
Bloque quirúrgico & 84 & 13 \\
Servicios centrales & 56 & 9 \\
Gestion/Serv. generales & 22 & 3 \\
Otro ámbito & 34 & 5 \\
Total & 644 & 100 \\
? & 32 & \\
& 676 & \\
\hline
\end{tabular}

\begin{tabular}{lrr}
\multicolumn{3}{c}{ Estado civil } \\
\hline Soltero & N & $\%$ \\
Casado & 367 & 26 \\
Divorciado & 61 & 10 \\
Viudo & 20 & 36 \\
Otro estado & 29 & 5 \\
Total & 636 & 100 \\
¿ & 40 & \\
& 676 & \\
\hline
\end{tabular}

\begin{tabular}{lrr}
\multicolumn{3}{c}{ Tipo de contrato } \\
\hline Fijo & N & \multicolumn{1}{c}{$\%$} \\
Vacante & 323 & 61,3 \\
Eventual & 139 & 26,4 \\
Total & 65 & 12,3 \\
¿? & 527 & 100,0 \\
& 149 & \\
& 676 & \\
& & \\
\hline
\end{tabular}

\begin{tabular}{lrr}
\multicolumn{3}{c}{ N. $^{\text {a }}$ de hijos } \\
\hline & $\mathbf{N}$ & $\%$ \\
\hline 0 & 147 & 29 \\
1 & 134 & 26 \\
2 & 175 & 34 \\
$>2$ & 52 & 10 \\
Total & 508 & 100 \\
$i ?$ & 168 & \\
& 676 & \\
\hline
\end{tabular}




\section{Descripción de los factores de la escala de satisfacción general}

El colectivo global de respondedores valoraron como satisfactorios, (puntuación media >4), seis factores: compañeros, superior jerárquico, responsabilidad asignada, variedad de tareas a realizar, horario y estabilidad en el empleo.

Moderadamente insatisfactorios (puntuación media 3-4) cinco factores: condiciones físicas de trabajo, posibilidad de aplicar un propio método de trabajo, reconocimiento, uso de la capacidad profesional y relación de profesionales con Dirección.

Y se apreciaron claramente insatisfactorias (puntuación media<3) cuatro factores: salario, posibilidades de promoción, atención a sugerencias y modo de gestión de la Organización.

El factor más satisfactorio fueron los compañeros y el menor el salario. Las siguientes tablas muestran la puntuación media en los factores de la encuesta de satisfacción general en el grupo global de respondedores y en los cinco grupos de profesionales con un número de respondedores significativo $(n>30)$.

Escala de Satisfacción Laboral $(\mathrm{n}=676)$

\begin{tabular}{lc}
\hline & Media \\
\hline Condiciones físicas de trabajo & 3,97 \\
Aplicar tu metodo de trabajo & 3,73 \\
Compañeros de trabajo & 5,11 \\
Reconocimiento de la tarea bien hecha & 3,78 \\
Con superior jerárquico & 4,07 \\
Con la responsabilidad asignada & 4,84 \\
Con el salario & 2,41 \\
Uso de la capacidad profesional & 3,77 \\
Relación profesionales-Dirección & 3,99 \\
Posibilidad de promoción & 2,70 \\
Modo de Gestión de la Organización & 2,58 \\
Atención a las sugerencias & 2,83 \\
Horario de trabajo & 4,03 \\
Variedad de tareas a realizar & 4,36 \\
Con la estabilidad en el empleo & 4,56 \\
\hline \multicolumn{2}{c}{ Media en los 15 ítems (rango 1 a 7) } \\
$\quad$ W de Kendall: 0,$38 ;$ p $<0,00005$ \\
\hline
\end{tabular}

Satisfacción Laboral en ATS/DUE (n=297)

\begin{tabular}{lc}
\hline & Media \\
\hline Condiciones físicas de trabajo & 4,14 \\
Aplicar tu metodo de trabajo & 3,98 \\
Compañeros de trabajo & 5,61 \\
Reconocimiento de la tarea bien hecha & 4,10 \\
Con superior jerárquico & 4,32 \\
Con la responsabilidad asignada & 5,01 \\
Con el salario & 2,54 \\
Uso de la capacidad profesional & 3,99 \\
Relación profesionales-Dirección & 4,18
\end{tabular}

Satisfacción Laboral en facultativos $\left(n=82^{\circ}\right)$

\begin{tabular}{lc}
\hline & Media \\
\hline Condiciones físicas de trabajo & 4,28 \\
Aplicar tu metodo de trabajo & 4,04 \\
Compañeros de trabajo & 4,85 \\
Reconocimiento de la tarea bien hecha & 3,56 \\
Con superior jerárquico & 3,82 \\
Con la responsabilidad asignada & 5,20 \\
Con el salario & 3,00 \\
Uso de la capacidad profesional & 4,38 \\
Relación profesionales-Dirección & 3,85 \\
Posibilidad de promoción & 3,01 \\
Modo de Gestión de la Organización & 2,94 \\
Atención a las sugerencias & 3,01 \\
Horario de trabajo & 4,35 \\
Variedad de tareas a realizar & 4,63 \\
Con la estabilidad en el empleo & 4,49 \\
\hline \multicolumn{2}{c}{ a Media de los 15 factores de la escala } \\
\hline
\end{tabular}

Satisfacción Laboral en Aux. Enfermería ( $\left.n=178^{\circ}\right)$

\begin{tabular}{lc}
\hline & Media \\
\hline Condiciones físicas de trabajo & 3,59 \\
Aplicar tu metodo de trabajo & 3,34 \\
Compañeros de trabajo & 4,74 \\
Reconocimiento de la tarea bien hecha & 3,48 \\
Con superior jerárquico & 3,96 \\
Con la responsabilidad asignada & 4,60 \\
Con el salario & 2,01 \\
Uso de la capacidad profesional & 3,39 \\
Relación profesionales-Dirección & 3,84
\end{tabular}




\begin{tabular}{lc}
\multicolumn{2}{c}{ Satisfacción Laboral en ATS/DUE (n=297) (cont.) } \\
\hline & Media \\
\hline Posibilidad de promoción & 3,01 \\
Modo de Gestión de la Organización & 2,73 \\
Atención a las sugerencias & 2,97 \\
Horario de trabajo & 4,01 \\
Variedad de tareas a realizar & 4,64 \\
Con la estabilidad en el empleo & 4,62 \\
\hline \multicolumn{2}{c}{ Media de los 15 factores de la escala } \\
\multicolumn{2}{c}{ W de Kendall: 0,$52 ; \mathrm{p}<0,00005$} \\
\hline
\end{tabular}

Satisfacción Laboral en celador $\left(n=43^{\circ}\right)$

\begin{tabular}{lc}
\hline & Media \\
\hline Condiciones físicas de trabajo & 3,02 \\
Aplicar tu metodo de trabajo & 2,74 \\
Compañeros de trabajo & 4,02 \\
Reconocimiento de la tarea bien hecha & 3,02 \\
Con superior jerárquico & 3,65 \\
Con la responsabilidad asignada & 4,28 \\
Con el salario & 2,19 \\
Uso de la capacidad profesional & 2,81 \\
Relación profesionales-Dirección & 3,33 \\
Posibilidad de promoción & 2,14 \\
Modo de Gestión de la Organización & 2,33 \\
Atención a las sugerencias & 2,51 \\
Horario de trabajo & 4,60 \\
Variedad de tareas a realizar & 3,65 \\
Con la estabilidad en el empleo & 4,28 \\
\hline \multicolumn{2}{c}{ a Media de los 15 factores de la escala } \\
$\quad$ W de Kendall: 0,$51 ; \mathrm{p}<0,00005$ \\
\hline
\end{tabular}

Satisfacción Laboral en Aux. Enfermería (n=178\%) (cont.)

\begin{tabular}{lc}
\hline & Media \\
\hline Posibilidad de promoción & 2,39 \\
Modo de Gestión de la Organización & 2,37 \\
Atención a las sugerencias & 2,66 \\
Horario de trabajo & 3,83 \\
Variedad de tareas a realizar & 4,02 \\
Con la estabilidad en el empleo & 4,46 \\
\hline \multicolumn{2}{c}{ a. Media de los 15 factores de la escala } \\
\multicolumn{2}{c}{ W de Kendall: 0,$42 ;$ p $<0,00005$} \\
\hline
\end{tabular}

Satisfacción Laboral en Administrativo ( $n=34$ )

\begin{tabular}{lc}
\hline & Media \\
\hline Condiciones físicas de trabajo & 4,24 \\
Aplicar tu metodo de trabajo & 3,88 \\
Compañeros de trabajo & 5,15 \\
Reconocimiento de la tarea bien hecha & 4,12 \\
Con superior jerárquico & 4,12 \\
Con la responsabilidad asignada & 4,59 \\
Con el salario & 2,09 \\
Uso de la capacidad profesional & 3,44 \\
Relación profesionales-Dirección & 4,18 \\
Posibilidad de promoción & 1,97 \\
Modo de Gestión de la Organización & 2,32 \\
Atención a las sugerencias & 2,74 \\
Horario de trabajo & 3,38 \\
Variedad de tareas a realizar & 4,03 \\
Con la estabilidad en el empleo & 4,62 \\
\hline \multicolumn{2}{c}{ Media en los 15 factores de la escala } \\
$\quad$ W de Kendall: 0,49; p $<0,00005$ \\
\hline
\end{tabular}

\section{Satisfacción laboral general}

Al valorar el grado de satisfacción general la mediana de puntuación total fue de 58 (rango de 15 a 102) que se corresponde con el intervalo neutro de satisfacción prefijado entre 52 y 67.

\begin{tabular}{lrr}
\multicolumn{3}{c}{ Grado de satisfacción laboral } \\
\hline & N & $\%$ \\
\hline Muy insatisfecho & 22 & 3,3 \\
Insatisfecho & 88 & 13,0 \\
Un poco insatisfecho & 168 & 24,9 \\
Neutro & 188 & 27,8 \\
Un poco satisfecho & 148 & 21,9 \\
Satisfecho & 59 & 8,7 \\
Muy satisfecho & 3 &, 4 \\
Total & 676 & \\
\hline
\end{tabular}

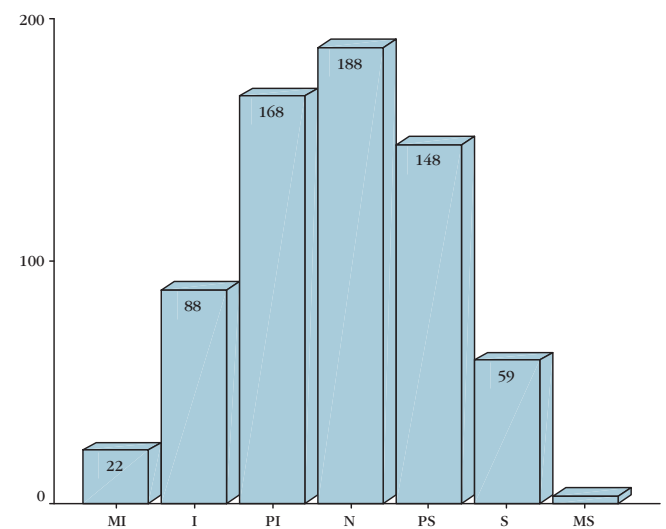


No se encontró diferencia significativa en la SL general por tipo de contrato $(\mathrm{p}=0,83)$, profesión (media en sanitarios de 57 frente a 54 en no sanitarios; $\mathrm{p}=0,13$ ), antigüedad en la profesión $(\mathrm{p}=0,79)$, antigüedad en el HUMV $(\mathrm{p}=0,51)$, número de hijos $(\mathrm{p}=0,84)$ y género (media de SL de 55,9 en varón frente a 57,3 en mujer; $\mathrm{p}=0,52$ ).

Se encontró diferencia significativa en SL por grupos de edad, siendo la media de SL menor en el grupo de 41 a 50 años $(p=0,01)$, por estado civil los grupos soltero y casado mostraron mayor grado de SL que el viudo, divorciado y otros $(\mathrm{p}=0,01)$, y por ámbito laboral $(\mathrm{p}=0,005)$, observándose tres grupos diferenciados, uno de relativa baja SL formado por profesionales de servicios centrales y del ámbito de Urgencias-UCI, un grupo intermedio de profesionales de plantas, bloque quirúrgico y servicios generales y un grupo de relativa mayor SL de profesionales de consultas externas.

Las siguientes tablas muestran la media y rango de SL percibida por las categorías de la tres variables que resultaron con relación significativa $(\mathrm{p}<0,05)$.

Satisfacción laboral por grupos de edad (Escala de 15 a 105)

\begin{tabular}{lrccr}
\hline & \multicolumn{4}{c}{ Satisfacción general } \\
\cline { 2 - 5 } & \multicolumn{1}{c}{ N } & Media & Mín. & Máx. \\
\hline $20-30$ & 38 & 55 & 22 & 93 \\
$31-40$ & 146 & 60 & 15 & 102 \\
$41-50$ & 179 & 53 & 15 & 92 \\
$51-60$ & 242 & 58 & 15 & 101 \\
$>60$ & 42 & 59 & 15 & 99 \\
Total & 647 & 57 & 15 & 102 \\
\hline $\mathrm{p}=0.01$ & & & &
\end{tabular}

Satisfacción laboral por estado civil

\begin{tabular}{lrccr}
\hline & \multicolumn{4}{c}{ Satisfacción general } \\
\cline { 2 - 5 } & \multicolumn{1}{c}{ N } & Media & Mín. & Máx. \\
\hline Soltero & 167 & 57 & 19 & 92 \\
Casado & 359 & 59 & 15 & 102 \\
Divorciado & 61 & 53 & 15 & 89 \\
Viudo & 20 & 52 & 15 & 83 \\
Otro & 29 & 52 & 15 & 93 \\
Total & 636 & 57 & 15 & 102 \\
\hline p=0.01 & & & &
\end{tabular}

Satisfacción laboral y ámbito profesional prevalente

\begin{tabular}{lrccr}
\hline & \multicolumn{4}{c}{ Satisfacción general } \\
\cline { 2 - 5 } & N & Media & Mín. & Máx. \\
\hline Plantas & 299 & 58 & 15 & 102 \\
Consultas & 67 & 64 & 15 & 99 \\
Urgencias/UCI/UARH & 82 & 53 & 15 & 87 \\
Bloque quirúrgico & 84 & 56 & 20 & 96 \\
Servicios centrales & 56 & 53 & 15 & 94 \\
Gestion/Serv. generales & 22 & 58 & 15 & 90 \\
Otro ámbito & 34 & 57 & 15 & 94 \\
Total & 644 & 57 & 15 & 102 \\
\hline p=0.005 & & & &
\end{tabular}

$\mathrm{p}=0.005$ 
Las siguientes tablas muestran la media y rango de la SL percibida por categorías de las variables que no resultaron relacionadas $(\mathrm{p}>0,05)$.

\begin{tabular}{lrccr}
\multicolumn{5}{c}{ Satisfacción laboral y tipo de contrato } \\
\cline { 2 - 5 } & \multicolumn{5}{c}{ Satisfacción general } \\
\cline { 2 - 5 } & N & Media & Mín. & Máx. \\
\hline Fijo & 323 & 58 & 15 & 96 \\
Vacante & 139 & 59 & 15 & 102 \\
Eventual & 65 & 58 & 19 & 92 \\
Total & 527 & 58 & 15 & 102 \\
& & & & \\
\hline
\end{tabular}

\begin{tabular}{lrccr}
\multicolumn{5}{c}{ Satisfacción laboral $\mathbf{y} \mathbf{n}^{\circ}{ }^{\circ}$ de hijos } \\
\cline { 2 - 5 } & \multicolumn{4}{c}{ Satisfacción general } \\
\hline 0 & 147 & 57 & 15 & 96 \\
1 & 134 & 59 & 16 & 102 \\
2 & 175 & 58 & 15 & 91 \\
$>2$ & 52 & 57 & 15 & 90 \\
Total & 508 & 58 & 15 & 102 \\
\hline
\end{tabular}

Satisfacción laboral y antigüedad en HUMV (años)

\begin{tabular}{lrccr}
\hline & \multicolumn{4}{c}{ Satisfacción general } \\
\cline { 2 - 5 } & N & Media & Mín. & Máx. \\
\hline$<4$ & 35 & 60 & 29 & 94 \\
$4-8$ & 117 & 58 & 15 & 92 \\
$9-12$ & 85 & 58 & 22 & 88 \\
$13-16$ & 59 & 57 & 16 & 102 \\
$17-25$ & 104 & 54 & 15 & 96 \\
$>25$ & 200 & 58 & 15 & 101 \\
Total & 600 & 57 & 15 & 102 \\
\hline
\end{tabular}

Satisfacción laboral por antigüedad en la profesión (años)

\begin{tabular}{lrccr}
\hline & \multicolumn{4}{c}{ Satisfacción general } \\
\cline { 2 - 5 } & \multicolumn{1}{c}{$\mathbf{~ N}$} & Media & Mín. & Máx. \\
\hline$<4$ & 18 & 57 & 23 & 93 \\
$4-8$ & 51 & 57 & 19 & 92 \\
$9-12$ & 98 & 56 & 15 & 88 \\
$13-16$ & 84 & 56 & 16 & 85 \\
$17-25$ & 135 & 56 & 15 & 102 \\
$>25$ & 242 & 58 & 15 & 101 \\
Total & 628 & 57 & 15 & 102 \\
\hline
\end{tabular}

\section{Satisfacción laboral intrínseca}

Al valorar el grado de satisfacción con factores de la tarea se obtuvo una mediana de 27, considerando que la escala de valores oscila entre 7 y 49 cabe considerar un grado neutro de satisfacción. El histograma de distribución muestra una tendencia plana con sesgo hacia la izquierda, de distribución no normal $(\mathrm{p}<0,00005)$.

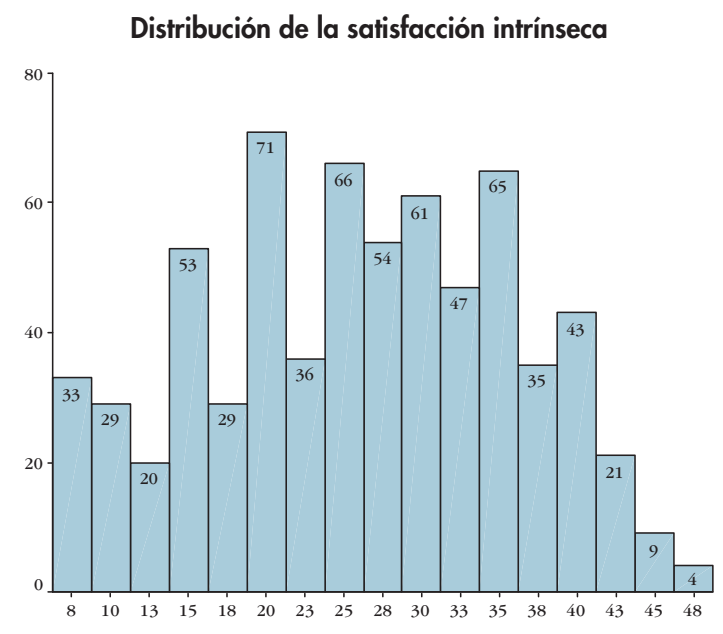

En el análisis bivariante la satisfacción intrínseca se relacionó con la profesión $(\mathrm{p}<0,0005)$ y edad $(\mathrm{p}=0,004)$. Se observa mayor satisfacción con la tarea en facultativos, DUE 
y técnicos de laboratorio, y menor en celadores y auxiliares de enfermería. Por edad mostró mayor satisfacción intrínseca en el grupo de 31 a 40 años.

No mostraron influencia significativa género, estado civil, tipo de contrato, número de hijos ni antigüedad en la profesión o en el HUMV.

Las siguientes dos tablas muestran la media y rango de SL intrínseca percibida por las categorías de las variables que resultaron con relación significativa $(\mathrm{p}<0,05)$.

\begin{tabular}{lrrrr}
\multicolumn{5}{c}{ Satisfacción laboral intrínseca por profesión } \\
\hline & \multicolumn{4}{c}{ Satisfacción intrínseca } \\
\cline { 2 - 5 } & \multicolumn{1}{c}{ N } & Media & Mín. & Máx. \\
\hline MÉDICO & 82 & 28 & 7 & 45 \\
DUE & 297 & 28 & 7 & 48 \\
AUX ENF. & 178 & 24 & 7 & 48 \\
CELADOR & 43 & 21 & 7 & 42 \\
ADMIN. & 34 & 25 & 7 & 44 \\
TEL. & 8 & 31 & 14 & 43 \\
OTRA & 16 & 27 & 7 & 40 \\
Total & 658 & 26 & 7 & 48 \\
\hline
\end{tabular}

\begin{tabular}{lrccc}
\multicolumn{5}{c}{ Satisfacción laboral intrínseca por grupo etario } \\
\hline \multirow{4}{*}{ Satisfacción intrínseca } \\
\cline { 2 - 5 } & N & Media & Mín. & Máx. \\
\hline $20-30$ & 38 & 26 & 7 & 42 \\
$31-40$ & 146 & 28 & 7 & 48 \\
$41-50$ & 179 & 24 & 7 & 44 \\
$51-60$ & 242 & 26 & 7 & 48 \\
$>60$ & 42 & 27 & 7 & 47 \\
Total & 647 & 26 & 7 & 48 \\
& & & &
\end{tabular}

Los siguientes gráficos muestran la distribución del grado de satisfacción en cada uno de los siete ítems cuya suma genera la subescala de satisfacción intrínseca.

La aplicación de método de trabajo propio

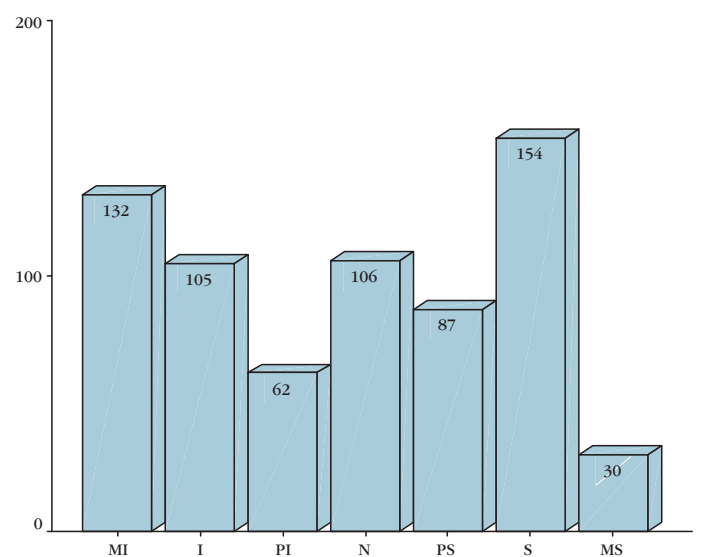

Con la responsabilidad asignada

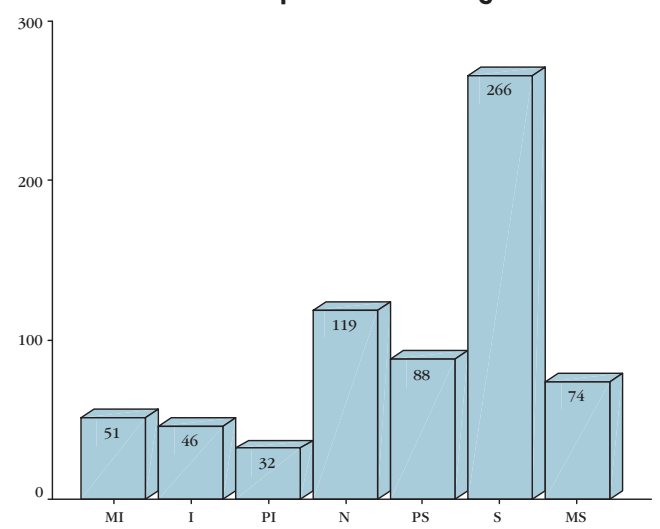

Reconocimiento

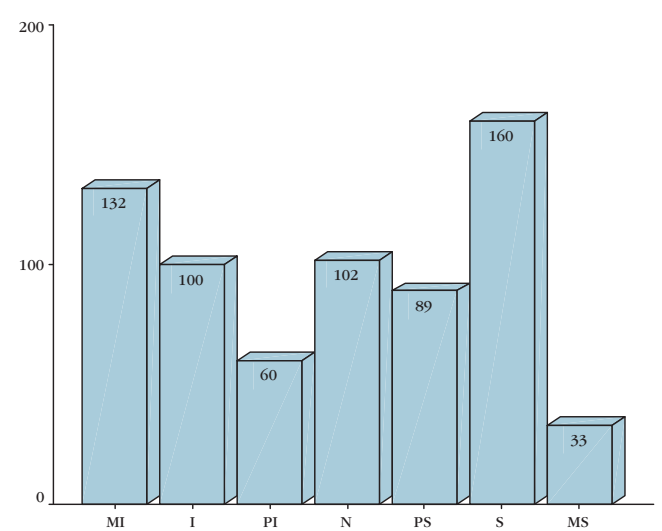

Con el uso de la competecia profesional

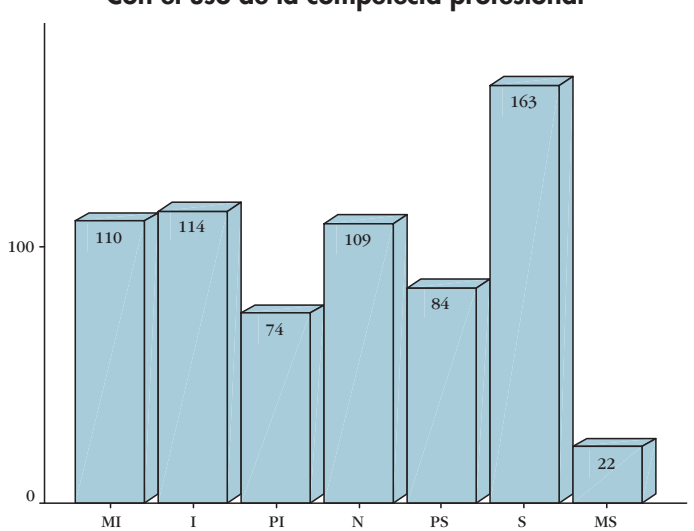



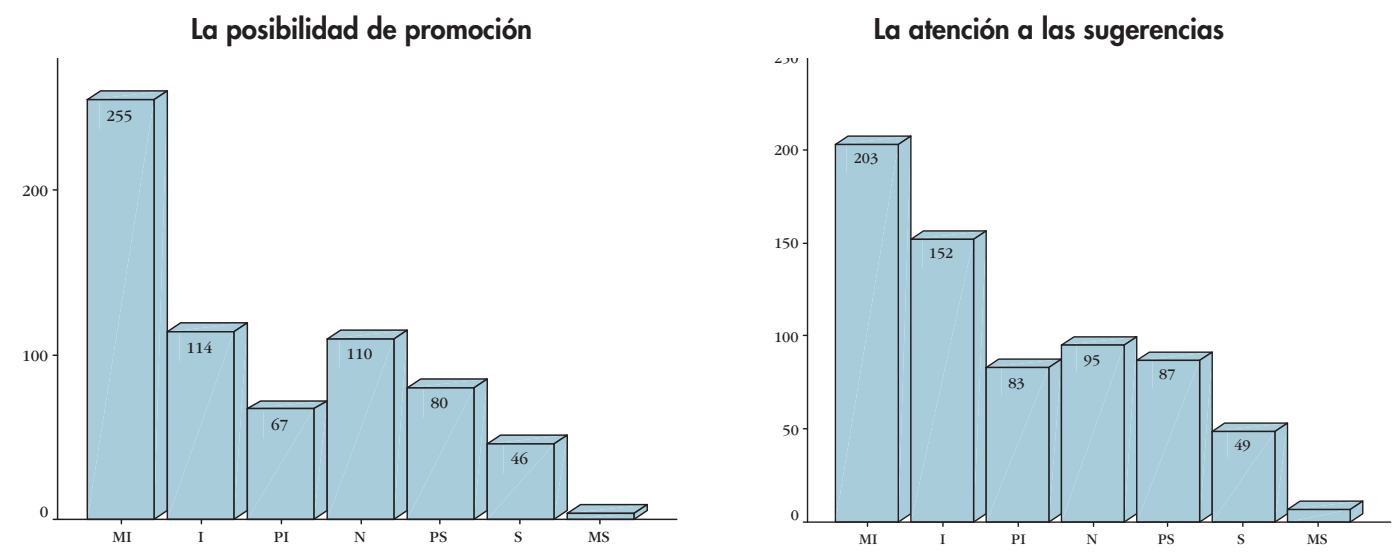

Con la variedad de tareas a realizar

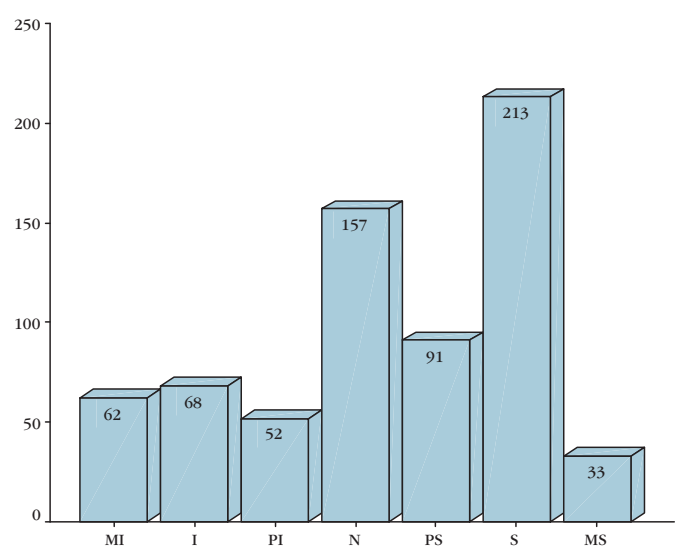

\section{Satisfacción laboral extrínseca}

Al valorar el grado de satisfacción extrínseca, o con factores ajenos a la tarea, se obtuvo una mediana de 31 . Teniendo en cuenta que la escala de valores oscila entre 8 y 56, cabe considerar un grado de satisfacción neutro. El histograma de distribución muestra una curva sesgada a la izquierda de distribución no normal $(\mathrm{p}<0,00005)$.

Distribución de la satisfacción extrínseca

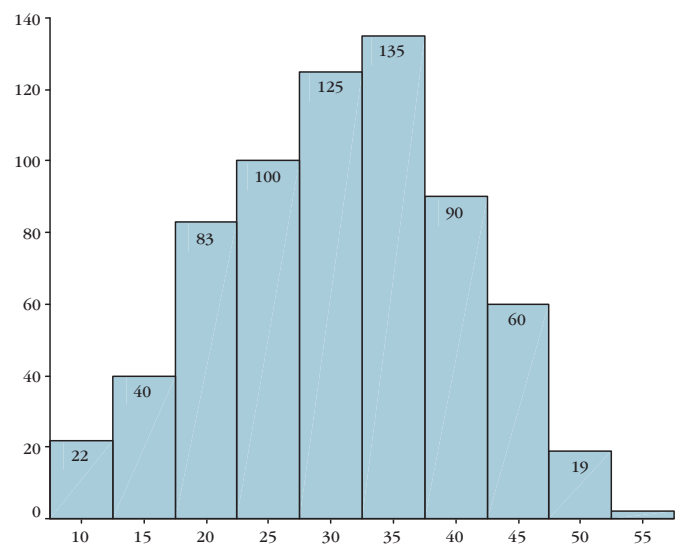

La satisfacción extrínseca se relacionó con la profesión $(\mathrm{p}<0,0005)$ y el ámbito laboral $(\mathrm{p}=0,004)$. Se observa mayor satisfacción extrínseca en facultativos, DUE, administrativos y técnicos de laboratorio, y menor en celador y auxiliar de enfermería. 
Por lugar de trabajo mayor satisfacción extrínseca mostraron trabajadores de consultas, plantas y bloque quirúrgico, mientras que menor los de UCI-Urgencias y servicios centrales. La edad tuvo una relación marginal $(\mathrm{p}=0,04)$.

No mostraron relación significativa género, estado civil, tipo de contrato, número de hijos ni antigüedad en la profesión o en el HUMV.

\begin{tabular}{lrrrr}
\multicolumn{5}{c}{ Satisfacción laboral extrínseca por ámbito laboral } \\
\cline { 2 - 6 } & \multicolumn{5}{c}{ Satisfacción extrínseca } \\
\cline { 2 - 6 } & 299 & 31 & 8 & 54 \\
Plantas & 67 & 34 & 8 & 52 \\
Consultas & 82 & 29 & 8 & 46 \\
Urgencias/UCIs & 84 & 30 & 13 & 51 \\
Bloque quirúrgico & 56 & 28 & 8 & 49 \\
Servicios centrales & 22 & 33 & 8 & 48 \\
Gestión/Serv. generales & 34 & 30 & 8 & 50 \\
Otro ámbito & 644 & 31 & 8 & 54 \\
Total & & & & \\
& & & &
\end{tabular}

Satisfacción laboral extrínseca por profesión

\begin{tabular}{lrcrr}
\hline & \multicolumn{4}{c}{ Satisfacción extrínseca } \\
\cline { 2 - 5 } & \multicolumn{1}{c}{$\mathbf{N}$} & Media & Mín. & Máx. \\
\hline MÉDICO & 82 & 32 & 8 & 51 \\
DUE & 297 & 32 & 11 & 54 \\
AUX ENF. & 178 & 29 & 8 & 53 \\
CELADOR & 43 & 27 & 8 & 49 \\
ADMINIST. & 34 & 30 & 8 & 49 \\
TEL. & 8 & 34 & 19 & 48 \\
PINCHE & 1 & 21 & 21 & 21 \\
OTRA & 15 & 36 & 8 & 48 \\
Total & 658 & 31 & 8 & 54 \\
\hline
\end{tabular}

Los siguientes gráficos muestran la distribución del grado de satisfacción en cada uno de los ocho ítems cuya suma genera la subescala extrínseca.

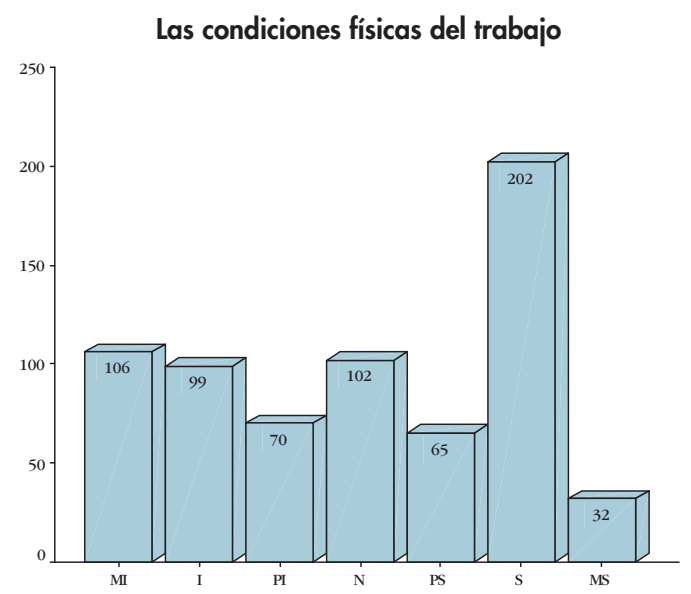

Los compañeros de trabajo
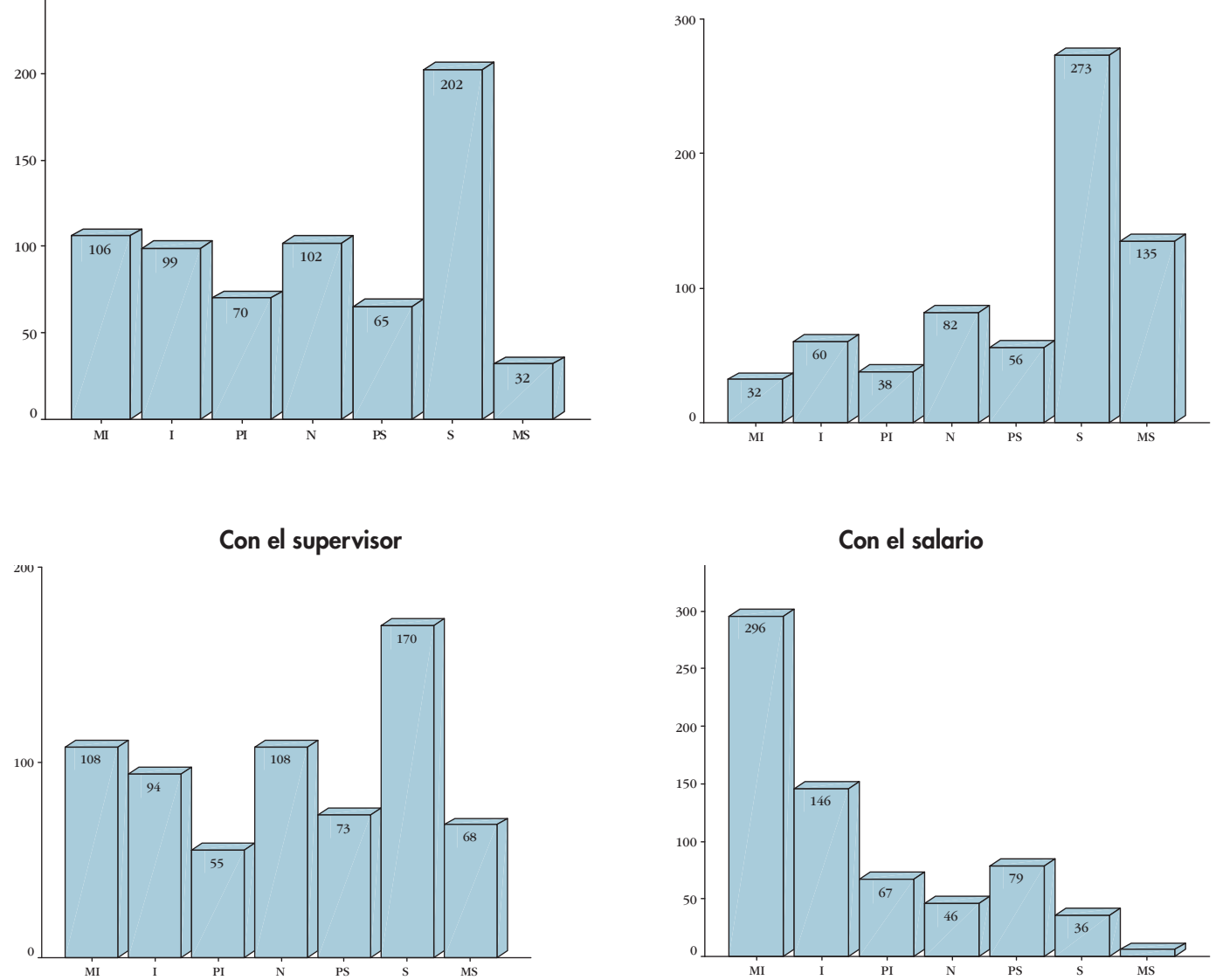


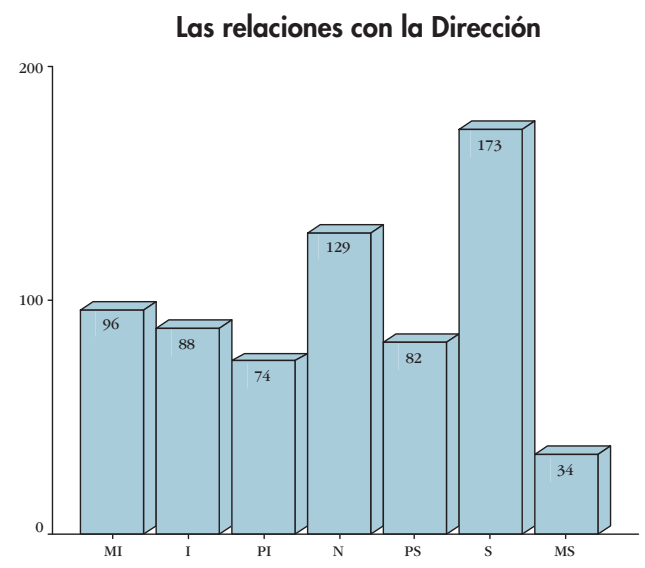

Con el horario de trabajo

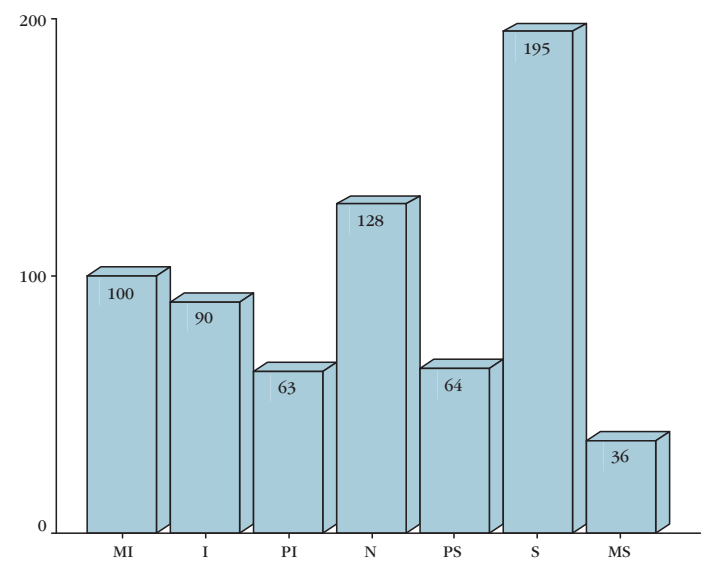

El modo de gestión de la organización

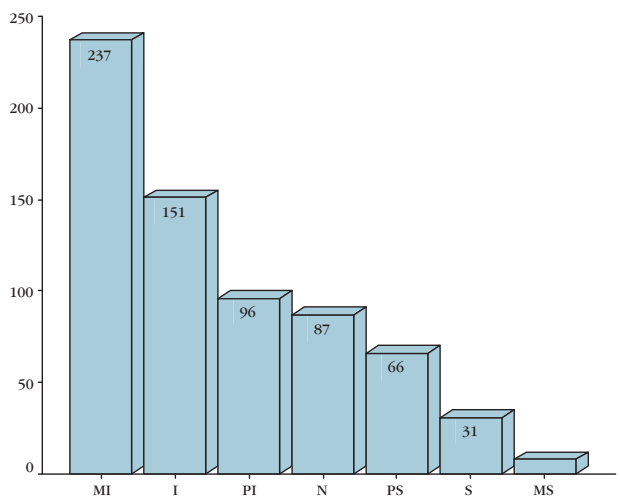

La estabilidad en el empleo

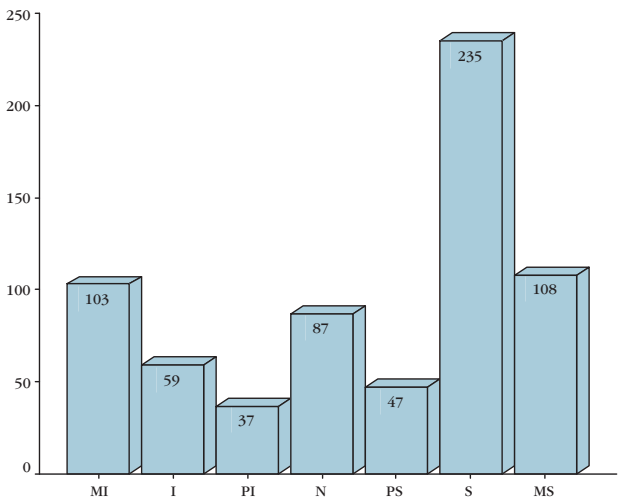

\section{Satisfacción con las personas}

Al considerar la subescala que valora la satisfacción con las personas de la Organización (compañeros, supervisores y dirección) se obtuvo una mediana de puntuación de 23. Teniendo en cuenta que la escala de valores oscila de 6 a 42 cabe considerar un grado neutro de satisfacción.

La satisfacción con las personas se relacionó con el ámbito laboral ( $\mathrm{p}<0,0005)$, profesión ( $\mathrm{p}=0,001)$ y edad $(\mathrm{p}=0,005)$. Así, por ámbito laboral se observó mayor satisfacción con las personas en trabajadores de consultas (media 26) y menor en los de servicios centrales (media 19). Por profesión fue mayor en técnicos de laboratorio (media 25) y menor en celadores (media 19), y por edad mayor en el grupo de 31-40 años (media 24) y menor en el de 41-50 (media 21).

Estado civil, género, número de hijos, tipo de contrato, antigüedad en la profesión ni antigüedad en el HUMV mostraron relación significativa. 
El siguiente gráfico muestra el histograma de distribución del grado de satisfacción con las personas, y las tablas su relación con las tres variables que resultaron con relación significativa: edad, profesión y ámbito laboral.

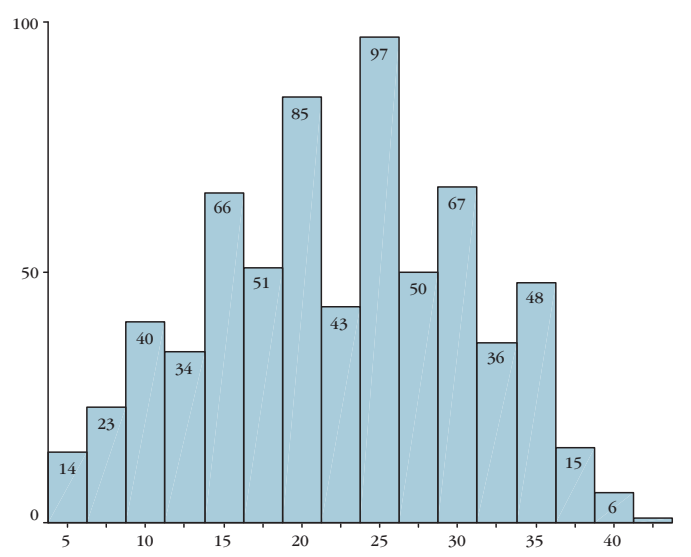

Satisfacción con las personas por grupos de edad

\begin{tabular}{lrccc}
\hline & \multicolumn{4}{c}{ Satisfacción con las personas } \\
\cline { 2 - 5 } & \multicolumn{1}{c}{ N } & Media & Mín. & Máx. \\
\hline $20-30$ & 38 & 22 & 7 & 39 \\
$31-40$ & 146 & 24 & 6 & 40 \\
$41-50$ & 179 & 21 & 6 & 37 \\
$51-60$ & 242 & 23 & 6 & 42 \\
$>60$ & 42 & 23 & 6 & 40 \\
Total & 647 & 23 & 6 & 42 \\
& & & & \\
& & & & \\
\hline
\end{tabular}

Satisfacción con las personas por profesión

\begin{tabular}{lrccc}
\hline & \multicolumn{4}{c}{ Satisfacción con las personas } \\
\cline { 2 - 5 } & \multicolumn{1}{c}{ N } & Media & Mín. & Máx. \\
\hline MÉDICO & 82 & 22 & 6 & 39 \\
DUE & 297 & 24 & 6 & 40 \\
AUX ENF. & 178 & 21 & 6 & 42 \\
CELADOR & 43 & 19 & 6 & 37 \\
ADMINIST. & 34 & 23 & 6 & 37 \\
TEL. & 8 & 25 & 11 & 37 \\
OTRA & 16 & 23 & 6 & 35 \\
Total & 658 & 22 & 6 & 42 \\
\hline
\end{tabular}

Satisfacción con las personas por ámbito laboral

\begin{tabular}{lcccc}
\hline & \multicolumn{4}{c}{ Satisfacción con las personas } \\
\cline { 2 - 5 } & N & Media & Mín. & Máx. \\
\hline Plantas & 299 & 23 & 6 & 42 \\
Consultas & 67 & 26 & 6 & 40 \\
Urgencias/UCI/UARH & 82 & 21 & 6 & 37 \\
Bloque quirúrgico & 84 & 22 & 6 & 38 \\
Servicios centrales & 56 & 20 & 6 & 37 \\
Gestion/Serv. generales & 22 & 23 & 6 & 35 \\
Otro ámbito & 34 & 22 & 6 & 37 \\
Total & 644 & 23 & 6 & 42 \\
\hline
\end{tabular}




\section{Apoyo social}

\section{Apoyo laboral instrumental}

Sobre una potencial puntuación comprendida en el rango de 0 a 9 , la mediana de este tipo de apoyo fue de 5 , lo que puede considerarse como una apreciación neutra de la utilidad de compañeros y supervisor inmediato ante dificultades de la tarea a realizar. No respondieron a este apartado 38 casos.

Distribución por apoyo instrumental (0 a 9)

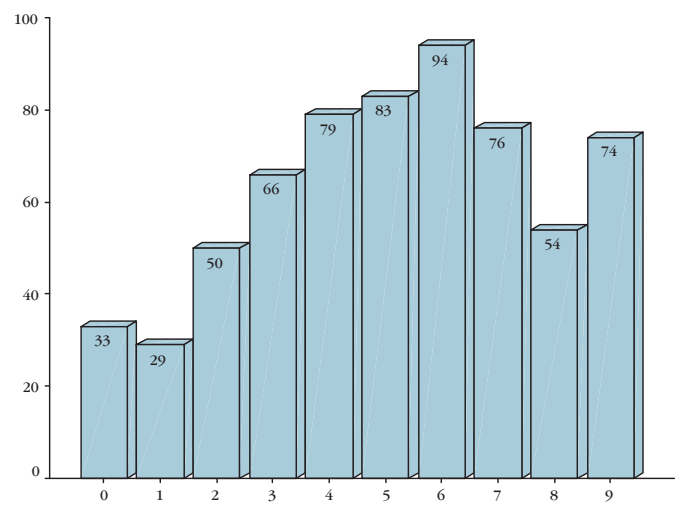

El apoyo instrumental se relacionó de forma directa con puntuaciones elevadas de los quince factores de la escala de satisfacción general. No obstante los ítems que mostraron un coeficiente de correlación más elevado fueron el grado de satisfacción con el superior jerárquico $(r=0,77 ; \mathrm{p}<0,00005)$ y con las relaciones con la dirección $(r=0,70$; $\mathrm{p}<0,00005)$. El coeficiente de correlación con el grado de satisfacción con compañeros de trabajo fue relativamente menor $(\mathrm{r}=0,50 ; \mathrm{p}<0,00005)$.

Los sanitarios perciben mayor ayuda instrumental que los no sanitarios (media de 5,3 frente a 4,$5 ; \mathrm{p}=0,01$ ). Por lugar de trabajo el apoyo instrumental se percibe más elevado en consultas, plantas y bloque quirúrgico y menor en servicios centrales $(\mathrm{p}<0,00005)$. La edad tuvo una relación marginal, con el grupo de 31 a 40 años como el que mayor apoyo instrumental percibía $(\mathrm{p}=0,05)$.

No mostraron relación sexo $(\mathrm{p}=0,3)$, estado civil $(\mathrm{p}=0,21)$, tipo de contrato $(\mathrm{p}=0,24)$ ni antigüedad en la profesión $(\mathrm{p}=0,85)$ o en el HUMV $(\mathrm{p}=0,53)$.

\section{Apoyo laboral emocional}

Sobre una potencial puntuación en el rango de 0 a 12, la mediana de apoyo fue de 9 , lo que puede considerarse como una apreciación media-alta de apoyo emocional en cuanto a la confianza que perciben los trabajadores para compartir los problemas del trabajo con compañeros y supervisor. No respondieron este apartado 43 casos (6\%).

Distribución por apoyo emocional (0 a 15)

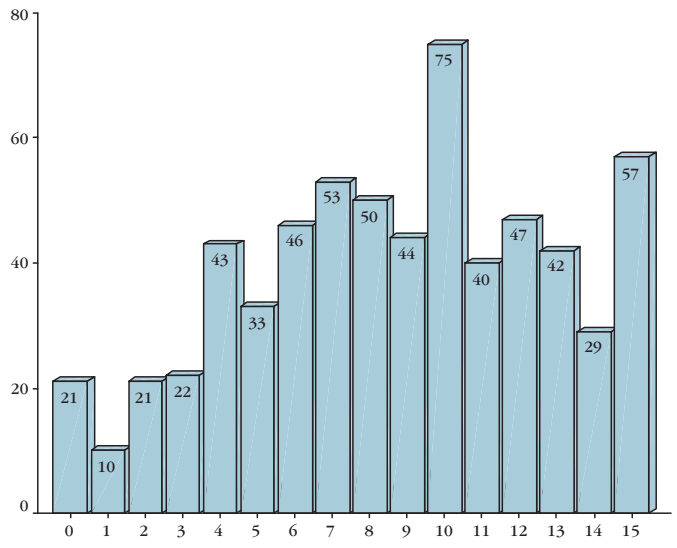


El apoyo emocional en el trabajo se relacionó de forma directa con puntuaciones elevadas de los quince factores de la escala de satisfacción general. No obstante los que mostraron un coeficiente de correlación mayor fueron el de satisfacción con el supervisor $(\mathrm{r}=0,75 ; \mathrm{p}<0,00005)$ y el de relaciones con la Dirección $(\mathrm{r}=0,69 ; \mathrm{p}<0,00005)$. De segundo orden en la relación fueron compañeros de trabajo $(r=0,56 ; p<0,00005)$, reconocimiento $(\mathrm{r}=0,55 ; \mathrm{p}<0,00005)$ y uso de la capacidad profesional $(\mathrm{p}=0,51 ; \mathrm{p}<0,00005)$.

No se observó asociación significativa con edad, sexo, estado civil ni antigüedad en la profesión o en el HUMV. El tipo de contrato mostró relación marginal $(\mathrm{p}=0,06)$, percibiendo mayor apoyo emocional de supervisor y compañeros el trabajador temporal que el fijo.

\section{Apoyo emocional no laboral}

Sobre una potencial puntuación comprendida en el rango de 0 a 12 , la mediana y moda de apoyo fue de 12, lo que puede considerarse como una apreciación alta de este tipo de apoyo que deriva del grado de confianza para compartir los problemas del trabajo con cónyuge, familiares o amistades. Si bien no respondieron este apartado 241 casos (36\%).

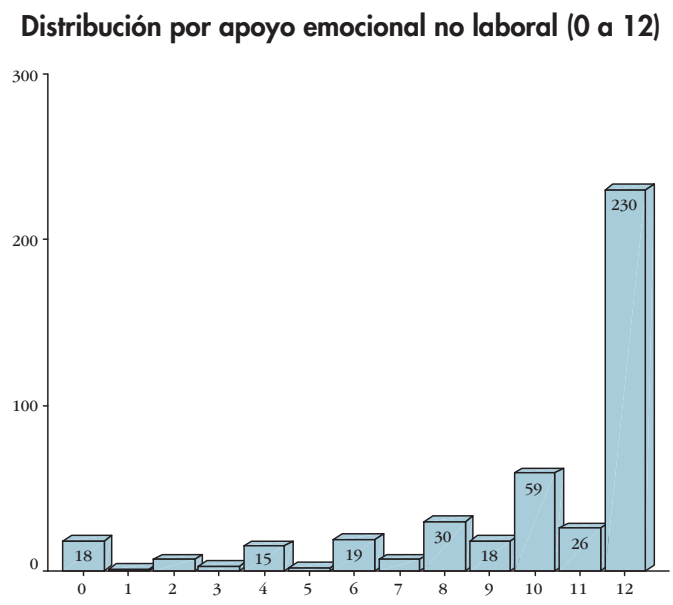

En el análisis bivariante la escala de apoyo emocional no laboral se relacionó de forma directa con el estado civil, puntuando más elevado los grupos de estado soltero y casado frente al de viudo o divorciado $(\mathrm{p}=0,007)$, y la edad $(\mathrm{p}=0,001)$, con el grupo $>60$ años y el grupo de 31 a 40 años con mayor puntuación media.

No se observó diferencia significativa por sexo, antigüedad en la profesión o en el HUMV, tipo de contrato y ámbito laboral. Cuando se estudió la correlación con los quince factores de la escala de satisfacción laboral general se encontró asociación significativa positiva, aunque débil, con las condiciones físicas o ambientales de trabajo, responsabilidad asignada, uso de la capacidad profesional y horario de trabajo $(r<0,2$; $\mathrm{p}<0,00005)$. 


\section{Relación entre satisfacción extrínseca, satisfacción intrínseca y apoyo social}

Se observó una correlación ordinal directa entre el grado de satisfacción en la subescala extrínseca y el grado de satisfacción en la subescala intrínseca $\left(\mathrm{p}<0,00005 ; \mathrm{r}_{\mathrm{o}}=0,84\right)$.

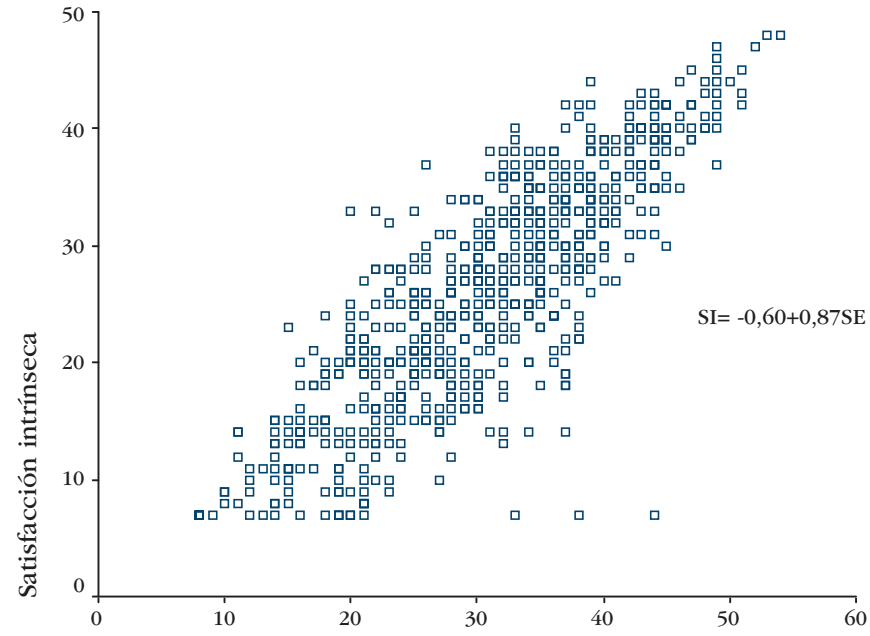

Así mismo, se observó una relación directa ordinal positiva entre satisfacción laboral $\mathrm{y}$ apoyo social en el trabajo, tanto emocional $\left(\mathrm{p}<0,00005 ; \mathrm{r}_{\mathrm{o}}=0,70\right)$ como instrumental $\left(\mathrm{p}<0,00005 ; \mathrm{r}_{\mathrm{o}}=0,69\right)$.

La escala de satisfacción general, sus tres subescalas (extrínseca, intrínseca, con las personas de la organización) y las dos escalas de apoyo social laboral (emocional e instrumental) mostraron importante correlación ordinal entre sí. Sin embrago, el apoyo no laboral emocional mostró un coeficiente de correlación débil o no resultó significativo.

La siguiente tabla muestra los coeficientes de correlación ordinal entre los diversos tipos de satisfacción laboral y de apoyo social.

\begin{tabular}{|c|c|c|c|c|c|c|c|}
\hline & SG & SE & SI & $\mathbf{S P}$ & EMLB & INLB & EMNLB \\
\hline \multirow{2}{*}{ Satisfacción general } & & ,948 & ,961 & ,920 &, 700 & ,698 & , 170 \\
\hline & &, 000 &, 000 &, 000 &, 000 &, 000 &, 000 \\
\hline \multirow{2}{*}{ Satisfacción extrínseca } & ,948 & & ,826 & ,891 & ,702 &, 702 & ,161 \\
\hline &, 000 & &, 000 &, 000 &, 000 &, 000 &, 001 \\
\hline \multirow{2}{*}{ Satisfacción intrínseca } & ,961 & ,826 & &, 867 & ,637 & ,638 & , 169 \\
\hline &, 000 &, 000 & &, 000 &, 000 &, 000 &, 000 \\
\hline \multirow{2}{*}{ Satisfacción con las personas } & ,920 & ,891 & ,867 & &, 771 &, 768 &, 104 \\
\hline &, 000 &, 000 &, 000 & &, 000 &, 000 &, 030 \\
\hline \multirow{2}{*}{ Apoyo emocional laboral } &, 700 &, 702 & ,637 &, 771 & &, 862 & ,107 \\
\hline &, 000 &, 000 &, 000 &, 000 & &, 000 &, 027 \\
\hline \multirow{2}{*}{ Apoyo instrumental laboral } & ,698 &, 702 & ,638 &, 768 & ,862 & & \\
\hline &, 000 &, 000 &, 000 &, 000 &, 000 & & \\
\hline \multirow{2}{*}{ Apoyo emocional no laboral } &, 170 &, 161 & ,169 & ,104 & ,107 & 076 & \\
\hline &, 000 &, 001 &, 000 &, 030 &, 027 & ,119 & \\
\hline
\end{tabular}

Primera fila: Coeficiente de correlación: rango de -1 (máxima inversa) y +1 (máxima directa).

Segunda fila: Se rechaza que la asociación detectada se deba al azar si p<0,005.

$S G=$ Satisfacción general; $S E=$ Satisfacción extrínseca; $S I=$ Satisfacción intrínseca; $\mathrm{SP}=$ Satisfacción con personas;

EMLB=Apoyo emocional laboral; INLB=Apoyo instrumental laboral; EMLB: = Apoyo emocional no laboral. 


\section{Análisis multivariante}

Mediante regresión logística se identificó el apoyo laboral emocional y el apoyo laboral instrumental como los dos factores con relación independiente con la SL general. El apoyo no laboral emocional tuvo relación marginal $(\mathrm{p}=0,05)$. Perdieron significación independiente edad, ámbito laboral y estado civil.

La siguiente tabla muestra la odss ratio de prevalencia (ORP) de los dos factores con relación independiente con la SL general y su intervalo de confianza.

\begin{tabular}{cccc}
\hline \multicolumn{4}{c}{$\begin{array}{c}\text { Satisfacción laboral general } \\
\text { Variables con relación independiente }\end{array}$} \\
\hline Variable & $\mathbf{p}$ & $\mathbf{O R P}$ & ORP $_{\text {Ic95 }}$ \\
\hline Apoyo instrumental & 0,0018 & 1,37 & $1,15-1.97$ \\
Apoyo emocional & 0,0001 & 1,51 & $1,16-1,61$ \\
\hline
\end{tabular}

El test Hosmer-Lemesow mostró bondad de ajuste $(\mathrm{p}=0,70)$. El modelo obtenido tiene una sensibilidad del $83 \%$ y especificidad del $79 \%$, clasificando de forma correcta el $83 \%$ de los sujetos estudiados.

\section{DISCUSIÓN}

La escala de Warr Cook y Wall (NTP 394; INSHT) genera el constructo de SL permitiendo valorar la experiencia afectiva de trabajadores por cuenta ajena en su trabajo. Tiene a su favor la consistencia, sencillez, facilidad de administración y estar validada por el INSHT para la evaluación de ciertos aspectos psicosociales del trabajo. A este respecto, estudios realizados por el Centro Nacional de Condiciones de Trabajo (CNCT-INSHT) señala que esta escala de SL se correlaciona con siete aspectos considerados en su método de evaluación de factores psicosociales: contenido del trabajo, carga mental, interés por el trabajador, definición de rol, relaciones personales, autonomía personal y calidad de la supervisión-participación. Es una escala que se diseñó a partir de estudios en trabajadores de la industria manufacturera inglesa y que ha demostrado su utilidad para valorar la SL en diversos entornos laborales ${ }^{17,18}$.

Como toda encuesta autoadministrada hay que tener en cuenta sus limitaciones en tanto que su base radica en apreciaciones subjetivas condicionadas por las expectativas de los propios respondedores sobre un conjunto de aspectos laborales. Así, Briner señala la dificultad de valorar de forma objetiva los factores psicosociales del trabajo a partir de cuestionarios autoadministrados al verse afectadas las respuestas por diversos factores ajenos a las condiciones de trabajo, y señala cuatro de estos factores como ejemplo. En primer lugar, el estado afectivo o emocional del respondedor al cumplimentar el cuestionario tiene considerable impacto en la forma en que aprecia los aspectos laborales que trata. Segundo, características propias del respondedor introduce sesgos en sus respuestas (personalidad, ansiedad, depresión, desmotivación). Tercero, la dificultad de distinguir entre verdaderas condiciones de trabajo insatisfactorias y evitables de las que resultan desagradables pero necesarias como la actividad jerarquizada o nocturna. Y cuarto, el hecho de que en ocasiones no se correlaciona la valoración subjetiva del trabajador de sus condiciones de trabajo con la evaluación técnica realizada en base a criterios científicos o legales ${ }^{19}$. Hoy se considera que la opinión del trabajador debe tenerse en cuenta al evaluar los aspectos psicosociales del trabajo pero no constituye la propia evaluación de riesgos psicosociales, de forma similar que el médico recoge los síntomas pero éstos no equivalen al diagnóstico ${ }^{20}$. Aun así, los cuestionarios autocumplimentado siguen siendo apreciados como un instrumento válido y clave para recabar las vivencias y percepciones personales de los trabajadores en sus condiciones de trabajo. 
En el presente estudio los trabajadores perciben en promedio un grado neutro de satisfacción laboral, situado en la banda de indiferencia. Este hallazgo es similar a lo encontrado por otros tanto en el ámbito hospitalario como en el de atención primaria ${ }^{12,21,22}$.

No se encontró diferencia en la SL percibida por género, ni en la escala general ni cuando se comparó por cada uno de sus quince factores. Estos datos contrastan con lo señalado por otros que encuentran mayor SL en la mujer ${ }^{23,24}$, concluyendo que se debe bien a que espera menos del trabajo, bien a un distinto esquema valorativo. Para estos autores la mujer, valoraría más las relaciones personales mientras que el hombre valoraría más la carrera profesional y el dinero ${ }^{25,26}$. No obstante, la relación entre género y SL no está bien esclarecida y no se ha encontrado en este estudio.

La observación de la tendencia al incremento de la SL con la edad coincide con lo señalado por otros, que concluyen que a medida que aumenta la edad del trabajador éste se muestra más satisfecho para implicarse en su tarea ${ }^{27}$. Algunos encuentran además relación directa entre edad y SL, con un máximo de SL en el período previo a la jubilación ${ }^{24}$. Concluyen que es probable que los trabajadores jóvenes muestren menor SL que los veteranos por estar al comienzo de su carrera profesional en tareas con peores condiciones laborales $^{28}$. Por último, otros han descrito una evolución en U de la SL en función de la edad, siendo mayor en jóvenes y veteranos y menor en una edad media ${ }^{29}$. Lo encontrado en este estudio también concuerda con esta observación.

No se encontró diferencia en SL por antigüedad en el hospital lo que concuerda con otros estudios $^{29}$. Sin embargo, otros sí han encontrado relación positiva entre antigüedad y satisfacción laboral, aunque en esta caso no puede descartarse la introducción de un sesgo de prevalencia al tender a abandonar la organización hacia otro ámbito sanitario los insatisfechos en fases tempranas ${ }^{6}$.

Al considerar los quince factores de la escala de SL se apreciaron más insatisfactorios cuatro: salario, posibilidades de promoción, atención a sugerencias y modo de gestión de la Organización. En la teoría bifactorial de Herzberg estos factores son higiénicos o extrínsecos a la actividad encomendada, si no que se relacionan con las condiciones en que ésta se desarrolla ${ }^{4}$. Son condiciones ajenas a la tarea encomendada administradas y decididas por la Dirección, por lo que los factores higiénicos están fuera del control del trabajador.

En situación de crisis económica es difícil la mejora salarial a corto plazo. Tampoco es previsible una mejora general de las posibilidades de promoción profesional en una estructura jerarquizada, pero al menos debe cuidarse que se lleve a cabo en base a una política transparente de equidad, mérito y capacidad ${ }^{30,31}$. Se fomentará que el trabajador aporte opiniones aunque dejando claro que a veces pueden ser poco prácticas o inviables; y el trabajador debiera ajustar sus expectativas comprendiendo que sus sugerencias serán escuchadas y razonadas pero no siempre podrán ser implantadas ${ }^{1}$. Otro aspecto a mejorar sería la comunicación efectiva y razonada en la toma de decisiones de gestión a nivel organizativo, incluso de las negativas que se juzgan necesarias de recorte de ventajas laborales invocando la crisis económica. A este respecto, decisiones recientes des te tipo han podido influir en la baja SL con el modo de gestión de la Organización.

Una valoración que ha resultado positiva a nivel general y por colectivo profesional evaluado ha sido la satisfacción con compañeros de trabajo. Éste es uno de los factores de mayor protección frente al estrés laboral. Una buena relación interpersonal aumenta el grado de SL e ilusión por el trabajo. En contrapartida, una mala relación es un factor de riesgo de desgaste profesional ${ }^{32}$.

La satisfacción con los jefes inmediatos se percibe en promedio de forma neutra o indiferente. Es mejor apreciada en los profesionales de enfermería y en grupo administrativo y de forma moderadamente insatisfactoria en facultativos, auxiliar de enfermería y personal celador. En este tipo de valoraciones subjetivas hay que tener presente el sesgo de relación personal al no tener esta porque corresponderse con una adecuada la relación profesional supervisor-trabajador. 
Llama la atención que la estabilidad en el empleo no se haya relacionado con la SL al no encontrar diferencia entre el personal fijo y el eventual. Otros autores, aunque en otro ámbito laboral, sí han encontrado diferencias entre ambos colectivos ${ }^{33}$.

Diversos estudios señalan una correlación entre baja satisfacción laboral y daño psicosomático $^{34}$. Es muy frecuente que tras síntomas físicos atribuidos al trabajo, sin causa evidente o proporcional, se encuentre malestar e insatisfacción con el puesto de trabajo $^{35}$. Así, una forma frecuente de manifestarse la insatisfacción laboral es la demanda de un puesto alternativo. Estos aspectos, evaluados por otros, no han sido considerados en el presente estudio ${ }^{8}$.

Acorde con la teoría empírica de Herzberg el presente estudio encontró fuerte relación entre la satisfacción extrínseca e intrínseca. Para Herzberg los factores extrínsecos son los que producen insatisfacción, mientras que la satisfacción laboral se debe a los factores intrínsecos. Herzberg, discípulo de Maslow, reformuló la teoría piramidal de éste por su teoría bifactorial ${ }^{4,36}$. Según Herzberg no se produciría la satisfacción por la tarea sin satisfacer en las condiciones de trabajo. Es una hipótesis lógica derivada de la observación empírica y de aplicación general. Sin embargo la teoría tiene excepciones, y en el mundo laboral siempre se han conocido casos de héroes satisfechos con su trabajo a pesar de unas condiciones insatisfactorias como en profesiones de elevado riesgo personal, trabajadores especialmente motivados, de organizaciones no gubernamentales o misioneros.

La relación observada entre el nivel de SL y las escalas que valoran el apoyo social laboral, afectivo e instrumental, fue estrecha. En menor grado con el apoyo social no laboral. La relación entre apoyo social en el trabajo y SL aparece por tanto evidente en este estudio. Sus resultados dibujan un profesional satisfecho en el trabajo que a la vez percibe un apoyo emocional e instrumental de compañeros y supervisor para el desarrollo de la tarea encomendada. Otros estudios también señalan la relación entre apoyo social y $\mathrm{SL}^{37,38}$.

Cada trabajador reacciona de manera diferente a los estresores laborales inevitables en base a sus habilidades y personalidad, y el apoyo social de compañeros y supervisor complementa sus recursos. Es conocido que el apoyo social en el trabajo protege frente al estrés, irritabilidad y quejas psicosomáticas ${ }^{39}$. También protege por efecto amortiguador (buffer) si el trabajador se expone a un estresor imprevisto o adicional de origen laboral (incidente violento con usuario) o vital (enfermedad grave ${ }^{40-42}$. En definitiva, el apoyo social tiene un impacto favorable en el mantenimiento de la salud física y mental ${ }^{43,44}$.

Se han distinguido cuatro tipos de apoyo social: emocional, instrumental, informativo y valorativo ${ }^{9,45}$. Y se ha señalado que se compone de cuatro factores: orientación directiva, ayuda no directiva, interacción social positiva y ayuda tangible ${ }^{46}$. De notar el gran valor del apoyo informativo recibido de una persona considerada significativa, como es un supervisor directo ${ }^{47}$.

Cabe destacar la importancia de la percepción del apoyo social, porque la realidad para cada trabajador es su realidad percibida. De modo que al percibir el apoyo identifica que es estimado y valorado, y que tiene a su disposición una red de comunicación y relación efectiva ${ }^{48}$.

Ante un acontecimiento estresante en el trabajo, el efecto amortiguador del apoyo social actúa moderando la respuesta emocional negativa, permitiendo reevaluarlo con una progresiva adaptación positiva ${ }^{47,49-51}$. Es así como el apoyo social contribuye a que trabajadores en fase de estrés o dificultad laboral superen el incidente y recuperen el equilibrio emocional en el trabajo.

No hay duda que existen otros factores no contemplados en este estudio que influyen en la $\mathrm{SL}^{52}$. Dado el amplio número de potenciales factores influyentes y la relevancia que tiene la SL para prestar un servicio sanitario de calidad ${ }^{53}$, es necesario abordar su mejora ${ }^{54}$. Aunque no hay receta universal, pues cada trabajador tiene sus propias necesidades y expectativas laborales, la teoría de Herzberg ofrece un punto de 
partida razonable para tratar los factores que condicionan la $\mathrm{SL}^{56}$. Según esta teoría se comenzará con los factores extrínsecos o higiénicos: política, supervisión, salario, relaciones interpersonales y condiciones de trabajo.

1. Las políticas de la organización serán equitativas y aplicables a todos los profesionales. Se evitarán las poco claras o innecesarias.

2. Se tendrá cuidado especial al establecer la línea jerárquica, dado el difícil papel del supervisor o mando intermedio. Tendrá aptitud de liderazgo, de trato respetuoso con el personal a su cargo y con capacidad de proporcionar apoyo emocional e instrumental cuando un trabajador a su cargo así lo requiera. Buen trabajador no es sinónimo de buen supervisor.

3. Facilitar la relación interpersonal disponiendo un tiempo por jornada para la socialización y generación del sentimiento de equipo de trabajo. Se promueve así el apoyo emocional e instrumental entre los trabajadores. Se evitará el trato rudo, prohibiendo conductas inapropiadas o comentarios ofensivos. Si un trabajador persiste en estos actos se tomarán medidas disciplinarias.

4. Todo trabajador aspira a un salario justo y equitativo, por lo que se seguirá una política transparente en salarios y complementos.

5. Las condiciones físicas del ambiente de trabajo tienen gran impacto en la SL. Se evitará trabajar con sensación de aglomeración y se hará lo posible por mantener el ambiente físico y los equipos de trabajo en buenas condiciones.

Una vez abordados los factores extrínsecos o higiénicos se tratarán los factores intrínsecos a la tarea: logro, reconocimiento, responsabilidad y promoción.

6. Se concienciará al trabajador que su tarea es importante para el buen hacer de la organización. Algunos pueden apreciar su tarea no interesante, pero se les debe transmitir que es indispensable para que el proceso sanitario resulte satisfactorio: un paciente tratado con tecnología de última generación puede percibir un mal servicio sanitario si el servicio de hostelería o el traslado en ambulancia a su domicilio ha sido defectuoso. Periódicamente se revisarán las tareas y cuando sea posible se simplificarán, o eliminarán las innecesarias.

7. Todo trabajador desea realizar bien su tarea, por lo que se ajustarán puestocompetencia profesional. No se fijaran objetivos imposibles, que abocan a la parálisis y frustración.

8. Las personas desean ser reconocidos por su trabajo. No es necesario un gran logro para prestar reconocimiento. Se concienciará al supervisor de la importancia de reconocer la tarea bien hecha y que ningún trabajador a su cargo se sienta ignorado. A nivel de Organización pueden fomentarse sistemas periódicos de reconocimiento público, como premios anuales.

9. El trabajador se siente satisfecho si dispone cierto grado de autonomía. Este aspecto es difícil de implantar en organizaciones sanitarias jerarquizadas o en las que se trabaja en equipo o en cadena. No obstante, donde sea posible se facilitará la autonomía para alcanzar los objetivos encomendados. A medida que el trabajador madura profesionalmente en su puesto, valorar la oportunidad de incrementarle la responsabilidad o de establecer nuevos objetivos desafiantes y significativos.

10. Considerar la promoción del trabajador de elevado rendimiento y competencia profesional. Si no hay un puesto jerárquico considerar la posibilidad de condecoración o premio especial por el desarrollo profesional alcanzado. Facilitar siempre la formación y la competencia profesional.

Por último, cabe considerar las limitaciones del presente estudio. Así, se ha realizado en una muestra no aleatoria de trabajadores que respondieron de forma voluntaria al cuestionario. Esto es susceptible al sesgo de selección que dificulta extrapolar los resultados a los trabajadores no respondedores. No obstante, cabe señalar que las variables de edad y sexo en la muestra son similares a los de plantilla del hospital. Otra 
limitación es la propia de los estudios de corte o transversales en cuanto a la dificultad de realizar inferencias causales a partir de la relación entre dos o más variables. Así, el grado de SL y el grado de apoyo social en el trabajo se han recogido al tiempo y aunque se detecta su asociación no es posible afirmar una dirección causal o si ambas son a la vez la consecuencia de una tercera causa no valorada, causa que en epidemiología de denomina factor de confusión.

\section{REFERENCIAS BIBLIOGRÁFICAS}

1. Ley 31/1995 de Prevención de Riesgos Laborales. BOE n. ${ }^{\circ}$ 269, de 10 de noviembre.

2. Real Decreto 39/1997 Reglamento de los Servicios de Prevención. BOE n. ${ }^{\circ} 27$ 31-01-1997.

3. Pérez J, Fidalgo M. NTP 394: Satisfacción laboral: escala general de satisfacción. INSHT (1994).

4. Herzberg F, Mausner B, Snyderman BB. The motivation to work. New York: John Wiley \& sons 1967.

5. Wallace JE, Lemaire JB, Ghali WA: Physician wellness: a missing quality indicator. Lancet 2009; 374: 1714-21.

6. Alonso I, Ayora JM, Bellás JA, Eloy J. Estudio de las posibles relaciones entre el absentismo laboral y el grado de insatisfacción profesional en la administración pública. Revista. Mapfre Medicina 1999; 10(1): 25-30.

7. Pathman DE, Konrad TR, Williams ES. Physician job satisfaction, dissatisfaction, and turnover. J Fam Pract. 2002; 51: 593

8. Mansilla F, García JC, Gamero C, Congosto A. Influencia de la insatisfacción laboral en las demandas de cambio de puesto de trabajo por motivos de salud. Med Segur Trab 2010; 56 (219): 147-157.

9. Pérez J, Martín F. NTP 439: El apoyo social. INSHT 1996.

10. Mansilla F, García JC, Gamero C, Congosto A, Calderón C, Gamero R. Abordaje de los trabajadores con trastornos físicos y psíquicos en salud laboral: un modelo. Med Segur Trab 2010; 56 (221): 274-279.

11. Goetz K, Campbell SM, Steinhaeuser J, Broge B, Willms S, Szecsenyi J. Evaluation of job satisfaction of practice staff and general practitioners: an exploratory study. BMC Family Practice 2011, $12: 137$.

12. López-Soriano F, Bernal L, Cánovas A. Satisfacción laboral de los profesionales en un Hospital Comarcal de Murcia. Rev Calidad Asistencial 2001; 16: 243-246.

13. Ramírez AJ, Graham J, Richards MA, et al. Mental health of hospital consultants: the effects of stress and satisfaction at work. Lancet 1996; 347: 724-8.

14. Harris MF, Proudfoot JG, Jayasinghe UW, et al: Job satisfaction of staff and the team environment in Australian general practice. Med J Aust 2007; 186: 570-3

15. Smith R. Why are doctors so unhappy? [editorial]. BMJ 2001; 322: 1073-4.

16. Dealing with job dissatisfaction in medicine [editorial]. Lancet 2001; 357: 1377.

17. Warr PJ, Cook J, Wall T. Scales for the measurement of some work attitudes and aspects of psychological well-being. Journal of Occupational Psychology 1979; 52: 129-148.

18. Cook J, Wall T, Warr PJ. The experience of work. Academic Press 1981. London.

19. Briner RB. Improving stress assessment: Toward an evidence-based approach to organizational stress interventions. J Psychosom Res 1997; 43: 61-71.

20. Los errores en la evaluación de riesgos psicosociales en el trabajo: prevenir la confusión MAPFRESeguridad 2006; 103: 29-42.

21. Fernández MI, Villagrasa JR, Gamo MF, Vázquez J, Cruz E, Aguirre MV, et al. Estudio de la satisfacción laboral y sus determinantes en los trabajadores sanitarios de un área de Madrid. Rev Esp Salud Pública 1995; 69: 487-97.

22. Fernández MI, Moinelo A, Villanueva A, Andrade C, Rivera M, Gómez JM, et al. Satisfacción laboral de los profesionales de atención primaria del Área 10 del Insalud de Madrid. Rev Esp Salud Pública 2000; 74: 139-47.

23. Alonso P. Estudio comparativo de la satisfacción laboral en el personal de la administración. Revista Psicología del Trabajo y de las Organizaciones 2008; 24: 25-40.

24. Lucas Marín L. Una aproximación sociológica al estudio de la satisfacción laboral en el trabajo en la provincia de Santander. REIS. 1981; 13: 99-100.

25. Sloane PJ, Williams H. Job satisfaction, comparison earnings and gender. Labour 2000; 14: 473-501.

26. Groot W, Maassen van den Brink H. Job satisfaction, wages and allocation of men and women, TSER/STT Working Papers 1998. WP-10-98.

27. Robina Ramírez R. Condicionantes sociolaborales de los empleados públicos: motivación y satisfacción laboral en la Administración Regional de Extremadura. Tesis doctoral. Universidad de Extremadura 2002 
28. Quinn, RP, Staines GL, y McCculloug MR. Job satisfaction: Is there a Trend?. Washington: US Department of Labor 1974.

29. Clark, A.E., Oswald, A.J. y Warr, P.B. Is job satisfaction U-shaped in age?. Journal of Occupational and Organizational Psychology 1997; 69: 57-81.

30. Sousa-Poza A, y Henneberger F. Analyzing job mobility with job turnover intentions: An international comparative study. 7th Annual Meeting of Society of Labor Economists 2002. Baltimore, USA.

31. Sousa-Poza, A. y Sousa-Poza, AA. Taking another look at the gender/ job- satisfaction paradox. Kyklos 2002; 53: 135-152.

32. González P, Suberviola R, González JF. Prevalencia del síndrome de burnout o desgaste profesional en los médicos de atención primaria. Aten Primaria 1998; 22: 580-4.

33. Frías Azcárate, M.R. (2006). Estudio de satisfacción del profesorado en la Universidad Pública Española. Empiria: Revista de metodología de ciencias sociales 2006; 11: 175-204.

34. Martínez Plaza CA (2009). Estrés laboral y trastornos musculoesqueléticos (I). Gestión práctica de riesgos laborales: Integración y desarrollo de la gestión de la prevención 2009; 61: 38-46.

35. ADECCO. El control del absentismo y la rotación. Documento de las Jornadas organizadas por Adecco Training. http://www.adecco.es/_data/NotasPrensa/pdf/92.pdf. 2006.

36. Maslow AH. Motivation and personality. 2nd ed. New York: Harper and Bros Publishers, 1970.

37. Masfield PK, Lois L, Vicary JR, Koch PB. Social support and the job satisfaction of female clerical woekers. Journal of Employment Consuleing 29, 113-116.

38. Hurlbert JS. Social networks, social circles, and job satisfaction. Work and Occupations 1991; 18: $415: 430$.

39. Parkes K, Mendham CA, Von Rabenau C. Social support and the demand-discretion model of job stress: Tests of additive an interactive effects in two samples. Journal of Vocational Behavior. 1994; 44: 91-113.

40. Schwarzer, R, Leppin A. Social Support and Health: A meta-analysis. Psychology and Health 1989; 3: 1-15.

41. Sánchez E, Barrón A. Social psychology of mental health: the social structure and personality respective. The Spanish Journal of Psychology 2003; 6: 3-11.

42. Helgeson V, Cohen S. Social support and adjustment to cancer: reconciling descriptive, corretional, and intervention research. Health Psychology 1996; 15: 135-148.

43. Schwarzer, R. y Leppin, A. Social Support and Health: A Theoretical and Empirical Overview. Journal of Social and Personal Relationships 1991; 8: 99-127.

44. Fernández Peña R. Redes Sociales, apoyo social y salud. Revista de recerca i investigació. Periferia, 3 www.periferia.name, 2005.

45. House JJ. Work stress and social support. Reading MA: Addison-Wesley 1981.

46. Barrera M, Ainley SL. The structure of social support: A conceptual and empirical analysis. Journal of Community Psychology 1983; 11: 133-144

47. Gore S. Stress-Buffering functions of social support: An Appraisal and c1arification of research models. En B.S. Dohrenwend y B.P. Dohrenwend (Eds.). Stressful life events and their contexts. New York: Prodist 1981.

48. Cobb S. Social support as a moderator of Iife events. Psychosomatic Medicine 1976; 5 300-314.

49. Cohen S y Wills, TA (1985). Stress, social support, and the buffering hypothesis. Psychological Bulletin 1985; 98: 310-57.

50. El-Bassel N, Guterman N, Bargal D, Su KH. Main and Buffering Effects of Emotional Support on Job and Health-Related strains: A national Survey of Israeli Social Workers. Employee Assistance Quaterly 2002; 13 : 1-18.

51. Frese M. Social support as a moderator of the relationship between work stressors and psychological dysfunctioning: a longitudinal study with objective measures. Journal of Occupational Health Psychology 1998; 4: 179-192.

52. Smith R. Why are doctors so unhappy? [editorial]. BMJ 2001; 322:1073-4.

53. Mira JJ, Aranaz J. La satisfacción del paciente como una medida del resultado de la atención sanitaria. Medicina Clínica 2000; 114(3): 26-33.

54. Haas JS, Cook EF, Puopolo AL, Burstin HR, Cleary PD, Brennan TA. Is the profesional satisfaction of general internists associated with patient satisfaction? J Gen Intern Med 2000; 15(2):122-8.

55. Kaarna M. The importance of job satisfaction in hospital quality process. Nordic School of Public Health. http://www.nhv.se. 2207

56. Syptack JM, Marsland DW, Ulmer D. Job Satisfaction: Putting Theory into practice. Fam Pract Manag. 1999; 6(9): 26-30. 


\section{ANEXO 1}

\section{Escala de Satisfacción General}

(Wall, Cook y Warr; 1976)

\begin{tabular}{ccccccc}
\hline $\mathbf{0}$ & $\mathbf{1}$ & $\mathbf{2}$ & $\mathbf{3}$ & $\mathbf{4}$ & $\mathbf{5}$ & $\mathbf{6}$ \\
\hline $\begin{array}{c}\text { Muy } \\
\text { Insatisfecho }\end{array}$ & Insatisfecho & $\begin{array}{c}\text { Poco } \\
\text { Insatisfecho }\end{array}$ & Neutro & $\begin{array}{c}\text { Poco } \\
\text { satisfecho }\end{array}$ & Satisfecho & $\begin{array}{c}\text { Muy } \\
\text { satisfecho }\end{array}$ \\
\hline
\end{tabular}

Según la tabla anterior, señale en la columna dcha. su grado de satisfacción con los siguientes aspectos.

Si alguno no se corresponde exactamente, por favor asócielo con la condición más semejante de su puesto.

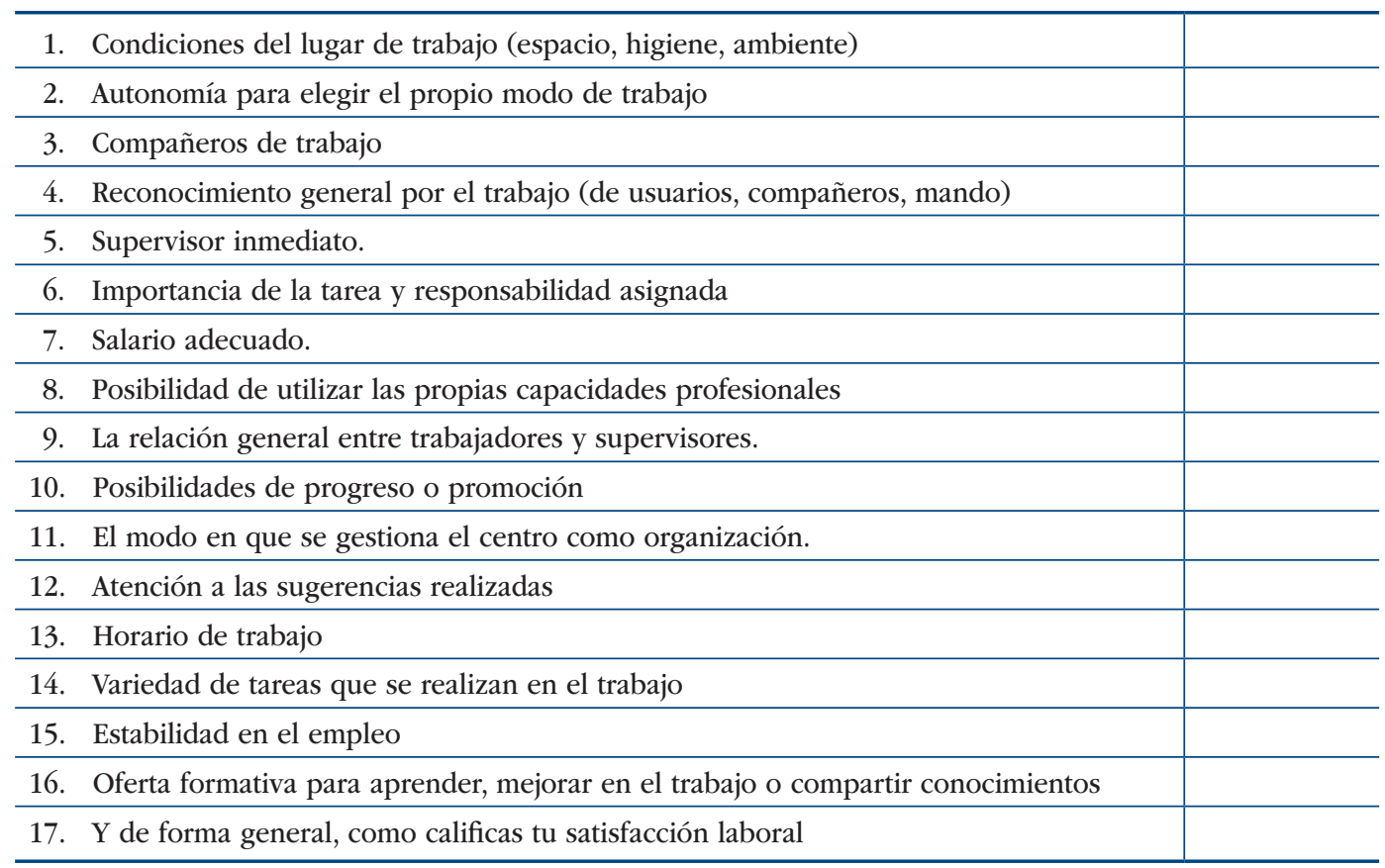




\section{ANEXO 2}

\section{Escala de Apoyo Social \\ (House y Wells; 1978)}

\begin{tabular}{cccc}
\hline $\mathbf{0}$ & $\mathbf{1}$ & $\mathbf{2}$ & $\mathbf{3}$ \\
\hline Nada & Poco & Algo & Mucho \\
\hline
\end{tabular}

Por favor, según la tabla anterior, señale en la columna derecha el grado

que corresponde al nivel de apoyo que percibe en los siguientes aspectos:

a. Ante un problema en el trabajo en qué grado puede confiar en las siguientes personas

\begin{tabular}{ll|l}
\hline 1. & Supervisor/Jefe inmediato. & \\
\hline 2. & Compañeros de trabajo. & \\
\hline 3. & Cónyuge. Si soltero/a señalar: o & \\
\hline 4. & Amigos o familiares. & \\
\hline b. & En qué grado las siguientes personas están dispuestas a escuchar sus problemas en el trabajo \\
\hline 1. & Supervisor/Jefe inmediato & \\
\hline 2. & Compañeros de trabajo. & \\
\hline 3. & Cónyuge. Si soltero/a marcar: o & \\
\hline 4. & Amigos o familiares. & \\
\hline c. & En qué grado le resultan útiles las siguientes personas para realizar tu trabajo & \\
\hline 1. & Supervisor/Jefe inmediato. & \\
\hline 2. & Compañeros de trabajo. & \\
\hline d. & En qué grado es cierto lo siguiente sobre su supervisor o jefe inmediato & \\
\hline 1. & Es competente en su trabajo, obtiene resultados del equipo. & \\
\hline 2. & Se preocupa por el bienestar de quienes están a su cargo. & \\
\hline
\end{tabular}

